\title{
Aufsatz
}

Bernhard Sicken

\section{Militärische Notwendigkeit und soziale Diskriminierung: Zur Ausweisung von Einwohnern aus preußischen Festungsstädten bei drohender Invasion (1830/31-1870/71)}

DOI 10.1515/mgzs-2015-0004

Zusammenfassung: Nach der Niederlage Preußens 1806/07, die das Heer drastisch zu schmälern zwang, rückten die Festungen zur Kompensation der numerischen Schwäche der Truppen stärker in den Vordergrund. Davon künden Richtlinien aus dem Jahr 1809, die für den Fall einer Einschließung und Belagerung langfristig u.a. die Requisition von Proviant und Baumaterial regelten. Auch die zivilen Bewohner der Festungsstädte fanden Beachtung, insoweit sie zu einer Belastung der Verteidigung zu werden drohten. Diese Gefahr begründete die Ausweisung der zur längeren Verproviantierung unfähigen Einwohner, für deren Unterhalt sich das Militär ebenso wenig wie für die Unterbringung im Umland verantwortlich fühlte, sondern die Aufgaben den Zivilbehörden zuschob. Die Versorgung der Abzuschiebenden warf große Probleme auf, da die maßgebliche lokale Daseinsvorsorge unzulänglich war. Letztlich erwies sich ein Ausquartieren von mehreren zehntausend Personen aus expandierenden Städten organisatorisch und politisch als unausführbar, zumal es in den sozioökonomisch schwierigen Ballungsräumen auch an Aufnahmekapazitäten mangelte. Darauf schränkte 1870 die Regierung informell die Ausweisungsbefugnis ein, doch blieb sie bis zum Ersten Weltkrieg rechtsverbindlich.

Schlüsselwörter: Militärisch-zivile Beziehungen, Festungsarmierung, Daseinsvorsorge, Städtewachstum, Belagerungsvorbereitung

Kontakt: Bernhard Sicken, Historisches Seminar, Domplatz 20-22, 48143 Münster, E-Mail:mgzredaktion@bundeswehr.org 
Das Verhalten der Zivilbevölkerung in bedrohten oder belagerten Festungsstädten hat in der Forschung trotz erheblicher militärischer Bedeutung für das 19. Jahrhundert bisher wenig Aufmerksamkeit gefunden. Diese Forschungslücke zum strukturbedingten Nebeneinander im Frieden, das bei einer Bedrohung mit ihren funktionalen Erfordernissen sowohl Züge eines Miteinanders als auch eines Gegeneinanders annehmen konnte, ist erstaunlich, weil es an Literatur zur Bau-, Architektur-, Technik- und Lokalgeschichte der mehrere Dutzend zählenden deutschen Festungen sowie an Studien zur Entwicklung der Befestigungsmanieren, zur strategischen Konzeption sowie zu Belagerungen nicht mangelt. Durchweg sind jedoch die Folgen des Festungsbaus und -unterhalts für die Bewohner, etwa von größeren Modernisierungen oder des Übergangs zur Kriegsbereitschaft, unbeachtet geblieben, obwohl hier Soldatisches und Bürgerliches enger als in einem herkömmlichen Garnisonort verflochten waren und eine derartige Konstellation von Kooperation, Distanz und Konfrontation den Gewinn weiterführender Erkenntnisse über die militärisch-zivilen Beziehungen zweifellos erleichtert. Kennzeichnet demnach ein historiografisches Defizit die eine Seite des hier zu behandelnden Anspruchs auf Ausweisung mittelloser und deshalb vermeintlich auch unzuverlässiger Städter zur Abwehr innerer Gefahr, so markiert das kontinuierliche Interesse der Streitkräfte an einer solchen Vorsorge die beinahe unbekannte andere Seite. Diese auf die ungünstige Quellenlage zurückzuführenden Lücken können erwartungsgemäß nicht durch die besser überlieferten normativen Regelungen geschlossen werden, die immerhin die Problematik im Bewusstsein der Amtsinhaber hielten und bei Bedarf ein rasches Vorgehen ermöglichten. Die den preußischen Festungsgouverneuren bzw. -kommandanten zugestandene Befugnis zum Entfernen Vermögensloser - über analoge Vorschriften in anderen Staaten ist nichts bekannt - wurde nach Ermessen gehandhabt; Vorbereitung und etwaiger Vollzug erfolgten unter Verantwortung der Zivilbehörden bei zögernder Mithilfe der überforderten und auf Diskretion bedachten Kommunen. Die Schwierigkeiten für eine fundierte Untersuchung spiegeln sich in den wenigen Vorarbeiten, die überwiegend der Stadtgeschichte mit anderen Schwerpunkten zuzuordnen sind. Zusammenfassende Darstellungen zur Thematik, geschweige denn systematisierende Analysen gibt es für das 19. Jahrhundert nicht, sondern nur verstreute, diachronisch auf ein oder zwei Bedrohungen beschränkte, mitunter wie zufällig anmutende Hinweise in Stadtmonografien, in Beiträgen zu Sammelbänden und Studien zur staatlich-bürokratischen Herrschaft. Erwähnung verdienen in erster Linie Arbeiten von Alf Lüdtke, Bernhard Sicken und Dieter KleinMeynen (siehe Anm. 2, 45, 74) mit substanziellen, wenngleich begrenzten Erkenntnissen. Zum spezifischen Untersuchungsgegenstand ist die Fragestellung in der Epoche der sich formierenden staatsbürgerlichen Gesellschaft noch nicht geworden. 
Grundlage des Beitrags sind - bedingt durch den Verlust des Heeresarchivs die einschlägigen, zum Teil lückenhaften Akten der Zivilbehörden, nämlich des preußischen Innenministeriums, der Oberpräsidien vor allem der Rheinprovinz und der Provinz Westfalen und diverser Regierungsbezirke. Hingegen wurde auf ein Auswerten kommunaler Archivbestände mit Ausnahme jener der Festung Glatz (heute Kłodzka) verzichtet, die der Überprüfung eines wichtigen, sich als fehlerhaft erweisenden Behördenbescheids diente, da die städtischen Zuständigkeiten weisungsgebunden waren und nur bei einer tatsächlichen Ausweisung größere Bedeutung erlangt hätten, sodass weiterführende Einsichten nicht zu erwarten waren. Quellen zur Wahrnehmung einer befürchteten Ausquartierung durch die breite Bevölkerung fehlen, punktuelle indirekte Hinweise erlauben nur dürftige Rückschlüsse.

1.

Die zwangsweise Entfernung von Einwohnern aus Festungsstädten, die bei einer Einschließung und Belagerung als Risiko eingeschätzt wurden, war lange Zeit gängige Empfehlung in den Kriegshandbüchern ${ }^{1}$, doch fand sie in größerem Umfang eher selten statt. Als Maxime wurde in Preußen an der vorsorglichen Ausweisung Unzuverlässiger, zu denen vor allem die Armen wegen fehlender Subsistenzmittel bei einer unter Umständen wochenlangen Zernierung zählten, aber durchgängig festgehalten. Dieser rigorose, auf eine ungehinderte Verteidigung zielende Anspruch, der schon in früheren Generationen hin und wieder durchgesetzt worden war, wurde nach dem Debakel von 1806/07 durch den Monarchen legalisiert, wie eine Instruktion für die Festungskommandanten mit der Bestätigung des Ausweisungsrechts bekundet ${ }^{2}$, der ergänzend Ende September 1809 eine Direktive für die höheren administrativen Dienststellen und die kommunalen Amtsinhaber folgte ${ }^{3}$, um die zivil-militärische

1 Siehe beispielsweise W[ilhelm] Rüstow, Die Lehre vom neueren Festungskrieg. Für Officiere aller Waffen bearbeitet, Bd 2, Leipzig 1860, S. 54-58.

2 Siehe Alf Lüdtke, »Gemeinwohl«, Polizei und »Festungspraxis«. Staatliche Gewaltsamkeit und innere Verwaltung in Preußen, 1815-1850, Göttingen 1982 (= Veröffentlichungen des Max-PlanckInstituts für Geschichte, 73), S. 272-274. Hier findet sich auch der Hinweis auf eine maßgebliche Denkschrift Neidhardts von Gneisenau aus dem Jahr 1808 und einen darauf basierenden Instruktionsentwurf; siehe dazu Die Reorganisation des Preußischen Staates unter Stein und Hardenberg. T. 2: Das preußische Heer vom Tilsiter Frieden bis zur Befreiung 1807-1914. Hrsg. von Rudolf Vaupel, Leipzig 1938, S. 733-735.

3 Landeshauptarchiv Koblenz (LHA Ko), Best. 403, Nr. 11311, S. 73-76. Ein Abdruck der Verfügung findet sich bei Volkmar Ulrich Meinhardt, Festung Minden. Gestalt, Struktur, Geschichte 
Zusammenarbeit unter selbstverständlicher Wahrung des Vorrangs der Befehlshaber sicherzustellen. Die als vertraulich eingestufte Anordnung ${ }^{4}$ für die zivile Seite trug pragmatische Züge und ließ konnotative patriotische Komponenten vermissen. Bekräftigt wurde hiermit die Befugnis der Kommandanten, Lieferungen zum Ausbau der Fortifikationsanlagen und zur Verpflegung auszuschreiben und notfalls einzutreiben, weshalb einige übereifrige Befehlshaber alsbald von den Zivilbehörden im Umland regelmäßige Angaben über die Lebensmittel- und Futtervorräte verlangten ${ }^{5}$, und überdies wurde ihnen nochmals das Recht zugesichert, "vor Eintritt der Einschließung alle diejenigen Personen, die wegen ihres Benehmens der Vertheidigung nachtheilig werden dürften oder sich für die Dauer der Belagerung nicht hinreichend verproviantiren können, aus der Festung zu entfernen « ${ }^{6}$. Die militärischem Ermessen überlassenen Zwangsmaßnahmen, die im Übrigen die politisch und gesellschaftlich »nachtheiligen« Individuen durchweg übergingen, trugen vordergründig subsidiäre Züge, denn ihnen war die Erlaubnis für jeden Bewohner zum rechtzeitigen Verlassen der Festung vorangestellt, der sich den Belastungen einer eventuellen Belagerung nicht gewachsen fühlte ${ }^{7}$.

Die Zeit vorsorglicher Verproviantierung, die von den Festungsbefehlshabern analog zum Truppenbedarf bei akuter Gefahr vorgeschrieben wurde ${ }^{8}$, schwankte zwischen drei und sechs Monaten und stellte somit unterschiedliche finanzielle Ansprüche an die Betroffenen. Von der Halbierung des Bevorratungsumfangs auf drei Monate, die für kleinere Fortifikationen schon 1831 gefordert ${ }^{9}$ und in diesem Jahr in Köln auch als Berechnungsgrundlage angesetzt wurde ${ }^{10}$, ist generell bis zum Jahr 1840 auszugehen; die Anpassung an ein realitätsnäheres Kriegsbild

einer Stadtfestung, Minden 1958 (= Mindener Beiträge, 7), S. 114-116, sowie ein knapper Verweis bei Manfred Böckling, Frauen in preußischen Festungen. Denkanstöße gesammelt am Beispiel der preußischen Garnison und Festung Koblenz und Ehrenbreitstein (1814-1918). In: Leben in und mit Festungen, Regensburg 2010 (= Festungsforschung, 2), S. 63.

4 Geheimes Staatsarchiv Preußischer Kulturbesitz Berlin (GStA PK), I. HA Rep. 77 Ministerium des Innern, Tit. $330^{\mathrm{a}}$, Nr. 18, Bd 1, Marienwerder, 17.1.1810. Bei fehlender Paginierung oder Foliierung der Archivalien wird zur näheren Kennzeichnung des benutzten Schriftstücks das Ausstellungsdatum angegeben.

5 Wie vorstehender Archivbeleg, hier Düsseldorf, 12.8.1821, Berlin, 29.11.1821.

6 LHA Ko, Best. 403, Nr. 11311, S. $74 \S 7$.

7 Ebd., S. $74 \S 5$.

8 Walter Dumbsky, Die deutschen Festungen von 1871 bis 1914: Strategische Bedeutung und technische Entwicklung, Frankfurt a.M. [u.a.] 1987 (= Erlanger Historische Studien, 11), S. 21, spricht in diesem Zusammenhang von einer »ökonomischen Armierung «.

9 LHA Ko, Best. 403, Nr. 1807, S. 163 ff. Rüstow, Die Lehre vom neueren Festungskrieg (wie Anm. 1), S. 54, geht im Allgemeinen von einer viermonatigen Verproviantierung aus.

10 LHA Ko, Best. 403, Nr. 1807, S. 357 ff. 
erfolgte offenbar Zug um Zug im pragmatischen Vorgehen. Doch diese Ermäßigung bedeutete für die von der Hand in den Mund Lebenden keine große Erleichterung und begünstigte nur die graduell Bessergestellten, denen unter Umständen sogar eine kommunale oder staatliche Beihilfe gewährt werden sollte ${ }^{11}$. $\mathrm{Zu}$ dieser Schicht rechneten vor allem jene Einwohner, denen dysfunktionales Verhalten nach amtlicher Einschätzung fernlag. Darüber hinaus konnten diejenigen auf ein Verbleiben hoffen, die Hilfe bei der Verteidigung »ihrer« Stadt erwarten ließen, sodass zu deren Versorgung selbst ein Rückgriff auf Militärmagazine als gerechtfertigt galt ${ }^{12}$. Grundsätzlich war der zivilen Seite an einer Beschränkung der Auszuweisenden gelegen, um die Evakuierung und Suche nach Ausweichquartieren zu erleichtern, zumal die Behörden für die Organisation ebenso wenig gerüstet waren wie für die materielle, überwiegend den Aufnahmegemeinden aufgehalste Versorgung ${ }^{13}$.

Erwartungsgemäß erwies sich die insgeheim vorgenommene Registrierung der aus materiellen Gründen eventuell Auszuweisenden als diffizil und erfolgte offenbar anhand lokaler Einschätzung von Bedürftigkeit, die teils vom Bezug von Armenunterstützung ausging, teils die Steuerveranlagung als Indikator zugrunde legte ${ }^{14}$. Punktuelle Angaben zur Bedürftigkeit nach der Jahrhundertmitte - frühere Erkenntnisse liegen anscheinend nicht vor - legen in grober Konturierung dar, dass in Festungsstädten mittlerer Größe pro Familie ein Tagessatz von mindestens einem halben Taler (Stand: 1851) ${ }^{15}$ bzw. 200 Taler (Stand: 1870) ${ }^{16}$ pro Jahr als Untergrenze galt, die vor einer schematisch angesetzten Evakuierung bewahrte, wobei freilich unklar bleibt, ob genauere Kenntnisse der örtlichen Amtsinhaber über die sozioökonomische Lage der Einwohner differenzierend wirkten und inwieweit etwaige Nachforschungen angesichts der als opportun angesehenen Diskretion der Ermittlungen möglich waren. Dass der Lebensunterhalt in einer

11 Ebd., S. 1 ff., 7 ff., und S. $55 \mathrm{ff}$.

12 Ebd., S. $55 \mathrm{ff}$. und S. 163ff., LHA Ko, Best. 403, Nr. 11311, S. $95 \mathrm{ff}$.

13 Siehe dazu in genereller Pointierung Dieter Langewiesche, "Staat« und »Kommune». Zum Wandel der Staatsaufgaben in Deutschland im 19. Jahrhundert. In: Historische Zeitschrift, 248 (1989), S. 623 und S. 629.

$14 \mathrm{Zu}$ den methodischen Problemen der Ermittlung repräsentativer Einkommen, dargelegt für Handwerker, doch strukturell übertragbar auf Angehörige anderer Schichten, siehe Karl Heinrich Kaufhold, Grundzüge des handwerklichen Lebensstandards in Deutschland im 19. Jahrhundert. In: Arbeiter im Industrialisierungsprozeß. Herkunft, Lage, Verhalten. Hrsg. von Werner Conze und Ulrich Engelhardt, Stuttgart 1979 (= Industrielle Welt, 28), S. 138f.

15 Landesarchiv Nordrhein-Westfalen, Abtlg. Rheinland, Duisburg (LAV NRW R), BR 0009, Nr. 1191, fol. 89f.

16 LHA Ko, Best. 403, Nr. 11311, S. $101 \mathrm{ff}$. 
größeren Stadt wie Köln kostspieliger war ${ }^{17}$ als in mittleren und vor allem kleineren Städten mit agrarischem Umland, ist naheliegend.

Die Absicht zur Verminderung unnützer Konsumenten nahm die Familien der Soldaten nicht aus, falls sie ihre Versorgung nicht sicherstellen konnten. Etwaige Zweifel bei den Befehlshabern, ob die Militärangehörigen zu dulden oder zu entfernen seien, wurden im Juli 1831 durch das Staatsministerium ausgeräumt, das generell die Ausquartierung anordnete, zugleich aber bestimmte, den vorübergehend »Exilierten« durch die Intendantur den üblichen Servissatz und die herkömmliche Brotportion zukommen zu lassen ${ }^{18}$. Für die Festung Minden, so sei als informatives Beispiel angeführt ${ }^{19}$, wurden daraufhin zwei Gemeinden und ersatzweise eine weitere als Ausweichort benannt, die in einer Entfernung von ca. einem Tagesmarsch lagen. Zum Abzug vorgesehen waren in diesem Jahr 194 Frauen und 422 Kinder $^{20}$, die überwiegend drei Garnisonkompanien ${ }^{21}$ mit ihren größtenteils älteren und nur eingeschränkt feldtauglichen Soldaten zuzuordnen waren.

Mühsamer und umstrittener war das Ermitteln geeigneter Evakuierungsgebiete für die Zivilbewohner. Den Regierungspräsidenten als subregional Verantwortlichen fiel diese Suche schwer, weil die Bereitschaft der aus amtlicher Sicht geeigneten, in der Nähe gelegenen Kreise und Orte gering war, für Wochen oder gar Monate fremden Leuten Unterkunft zu gewähren, die zur Bürde zu werden drohten. Nur zögernd konnten darum Ausweichgebiete, meist erst unter Nachdruck der aufsichtführenden Behörden festgelegt werden ${ }^{22}$. Es muss als bezeichnend für dieses problematische Bemühen betrachtet werden, dass im Rheinland wiederholt eine Beanspruchung westfälischer Kreise ins Gespräch gebracht wurde ${ }^{23}$, der insofern eine gewisse Berechtigung zuerkannt werden muss, als fünf Festungen mit

17 Siehe mit generellem Hinweis zu den Lebenshaltungskosten von Arbeitern und Handwerksgesellen Jürgen Herres, Köln in preußischer Zeit 1815-1871, Köln 2012 (= Geschichte der Stadt Köln, 9), S. 368 und S. 375, sowie resümierend Kaufhold, Grundzüge des handwerklichen Lebensstandards (wie Anm. 14), S. 160.

18 Landesarchiv Nordrhein-Westfalen, Abtlg. Ostwestfalen-Lippe, Detmold (LAV NRW OWL), M 1 Pr, Nr. 139, fol. 13.

19 Siehe mit weiteren Angaben zur Ausweisung der Militärbevölkerung in diesem Beitrag S. $106 \mathrm{ff}$.

20 LAV NRW OWL, M 1 Pr, Nr. 139, fol. 15.

21 Siehe ebd., M 1 Pr, Nr. 21, fol. 301v; hier werden für die Garnisonkompanien insgesamt 516 Frauen und Kinder genannt, von denen die Masse, die von der (Bezirks-)Regierung Minden als "unerschöpfliches Armen Depot« bezeichnet wurde, zur Ausweisung bestimmt war. Vgl. auch Curt Jany, Geschichte der Preußischen Armee vom 15. Jahrhundert bis 1914, Bd 4: Die Königlich Preußische Armee und das Deutsche Reichsheer 1807 bis 1914, Berlin 1933, S. 129 f.

22 Siehe LHA Ko, Best. 403, Nr. 1807, S. 99f. und S. 109f., ferner LAV NRW R, BR 0009, Nr. 1191, fol. $26 \mathrm{ff}$. und S. $36 \mathrm{ff}$.

23 LHA Ko, Best. 403, Nr. 1807, S. 149 f. 
einer Vielzahl bedürftiger Einwohner in der Rheinprovinz lagen, hingegen nur ein derartiges Bollwerk auf Westfalen entfiel und deshalb ein Ausgleich bei der Belastung erwägenswert war, zu dem sich die Provinz jedoch nicht bereitfand ${ }^{24}$, die sich bei ihrer Ablehnung auf Rückhalt beim Innenminister berufen konnte ${ }^{25}$.

Bei den Ausweisungsplänen stellte neben der Unterbringung, die mit ihren räumlichen Schwerpunkten den Festungsbefehlshabern anzuzeigen war $^{26}$ und Mitsprache ermöglichte, der Unterhalt das Hauptproblem dar, dessen Lösung das Kriegsministerium im Spätjahr 1831 flugs dem Innenministerium zugeschoben hatte ${ }^{27}$ und damit einem Ressort, das weder über ein fundiertes Konzept zur Übernahme der Aufgabe noch über die finanziellen Mittel für effektiven Beistand verfügte $^{28}$, sodass letztlich nur ein Verlagern der Verantwortlichkeit auf nachgeordnete Instanzen unter Rückgriff auf die Kommunen verblieb. Die Federführung hatten die Oberpräsidenten zu übernehmen ${ }^{29}$; diesen Spitzenbeamten, die ohnehin zur Unterstützung des Militärs verpflichtet waren und zugleich die Interessen ihrer Region zu wahren hatten ${ }^{30}$, fiel somit ein weiterer Auftrag koordinierender und beaufsichtigender Art zu. Ein Fonds zur Subventionierung überforderter Gemeinden und Staatsbürger blieb ihnen versagt ${ }^{31}$, obwohl nachgeordnete Behörden wiederholt um finanziellen Beistand nachgesucht hatten, um der $\mathrm{zu}$ erwartenden Not bei einer massenhaften Ausweisung zuvorzukommen ${ }^{32}$. Wie vorhersehbar war auch der Vorschlag zum Rückgriff auf die lokalen Erträge der Mahl- und Schlachtsteuer sogleich auf Ablehnung gestoßen ${ }^{33}$, weil einerseits eine

24 Ebd., S. 190.

25 Ebd., Nr. 11311, S. 7 f.

26 Siehe bspws. GStA PK, I. HA Rep. 77 Ministerium des Innern, Tit. 330 a , Nr. 18, Bd 1, Berlin, 27.3.1831.

27 LHA Ko, Best. 403, Nr. 11311, S. 5 f.

28 Ebd., S. 1f. und S. 7 f. Zur behördlichen Unsicherheit im Fall einer Bedrohung, hier artikuliert durch den Oberpräsidenten der Rheinprovinz, siehe auch GStA PK, I. HA, Rep. 77 Ministerium des Innern, Tit. 332 ${ }^{\mathrm{bb}}$, Nr. 4, Bd 1, Berlin, 13.4.1831.

29 Eine Oberpräsidentenliste findet sich im Anhang des Sammelbands Die preußischen Oberpräsidenten 1815-1945. Hrsg. von Klaus Schwabe, Boppard a.Rh. 1985 (= Deutsche Führungsschichten der Neuzeit, 15), S. 277-294.

30 Siehe Reinhart Koselleck, Preußen zwischen Reform und Revolution. Allgemeines Landrecht, Verwaltung und soziale Bewegung von 1791 bis 1848, 2. Aufl., Stuttgart 1975, S. 221-237, und ferner zu den Obliegenheiten der Oberpräsidenten Rüdiger Schütz, Die preußischen Oberpräsidenten 1815-1866. In: Die preußischen Oberpräsidenten (wie Anm. 29), S. 68-76, sowie Gesetz=Sammlung für die Königlichen Preußischen Staaten 1815, Berlin [1815], Nr. 9, S. 86, Gesetz=Sammlung 1817, Nr. 15, S. 230, 235f., 250 und S. 259, Gesetz=Sammlung 1826, Nr. 1, S. 3.

31 Siehe LAV NRW R, BR 0009, Nr. 1191, fol. 44f., ferner LHA Ko, Best. 403, Nr. 1807, S. 315 ff.

32 LHA Ko, Best. 403, Nr. 1807, S. 7 ff., und Nr. 11311, S. 7 f.

33 Ebd., Nr. 1807, S. 1ff. und S. 11. 
Zweckbindung von Steuern aus prinzipiellen Gründen verworfen wurde und andererseits die Anerkennung einer staatlichen Pflicht zur Unterstützung nicht in Betracht $\mathrm{kam}^{34}$, galt doch die Daseinsvorsorge traditionell als kommunale Aufgabe. Als Ergebnis der Sondierungen über etwaige Hilfen zeichnete sich eine kaum kaschierte Unsicherheit ab: So vertrat beispielsweise ein Regierungspräsident die Ansicht, die zur Aufnahme Ausgewiesener bestimmten Orte dürften keineswegs zum Beistand für deren Lebensunterhalt angehalten werden ${ }^{35}$, der folglich dem Geschick Bedürftiger seinen Lauf lassen wollte, oder von anderer Seite wurden die vermeintlichen Chancen zum Broterwerb am Evakuierungsort ${ }^{36}$ oder in anderen Gemeinden ${ }^{37}$ als wohlfeiler Ausweg angeführt, obschon alle Erfahrungen solchen Erwartungen widersprachen. Von der Überforderung der mittleren und unteren Behörden kündet das wiederholte Verweisen auf private Hilfsbereitschaft, die angeblich »von dem Patriotismus und dem Wohltätigkeits=Sinn des Landes $^{38}$ erwartet werden dürfe. Eine Lösung fand sich nicht, denn noch 1867 resümierte der schlesische Oberpräsident über geplante, jedoch nicht vollzogene Ausweisungen, zur Existenzsicherung Vermögensloser ${ }^{39}$ den Rückgriff auf Regelungen des Armengesetzes ${ }^{40}$ beabsichtigt zu haben, ferner auf disponible Gelder aus regionalen staatlichen Armenfonds gesetzt und nicht zuletzt auf private Mildtätigkeit gehofft zu haben. Das Rekurrieren auf notdürftige situative Aushilfen, die mehr schlecht als recht die Bedürfnisse zu decken vermochten, war nach wie vor kennzeichnend.

Ersichtlich wird die amtliche Unsicherheit bei den vorgesehenen Abschiebungen durch vage Richtlinien, deren Beachtung von vornherein als zweifelhaft einzuschätzen war. Hierzu gehörte das Rechnen auf militärischen Beistand beim Ausweisen von Städtern, von dem für die Verteidigung als nachteilig angesehene, doch nicht näher spezifizierte vermögende Bürger ebenfalls betroffen sein konnten ${ }^{41}$, und zählte zudem die angebliche Übernahme notwendiger Krankentransporte durch die zum Ausweichquartier bestimmten Orte ${ }^{42}$, die bei solchen Leistungen allerdings auf militärischen Vorspann hoffen durften. Immerhin

34 Siehe mit ausweichender Antwort des Innenministeriums ebd., Nr. 11311, S. $7 \mathrm{f}$.

35 Ebd., Nr. 1807, S. 1 ff.

36 Ebd., S. $109 \mathrm{f}$.

37 Ebd., Nr. 11311, S. 7 f.

38 Ebd., Nr. 1807, S. 145. Siehe mit ähnlichem Tenor auch LAV NRW R, BR 0009, Nr. 1191, fol. $44 \mathrm{f}$.

39 LHA Ko, Best. 403, Nr. 11311, S. 19. Siehe auch im vorliegenden Beitrag Anm. 104.

40 Siehe Gesetz=Sammlung für die Königlichen Preußischen Staaten 1843, Berlin [1843], Nr. 1, S. $12 \S 26$.

41 LHA Ko, Best. 403, Nr. 1807, S. 139 ff.

42 Ebd. 
zeichnete sich beim Vollzug von Ausquartierungen, die durch das Wachstum mancher Festungsstädte eine nicht vorhergesehene Dimension annahmen, schließlich für die verantwortlichen Behörden und die betroffenen Einwohner insofern eine Erleichterung ab, als das Kriegsministerium im Jahr 1866 eine kostenfreie Beförderung durch die Eisenbahn in Aussicht stellte ${ }^{43}$, weil ohne den Rückgriff auf dieses Transportmittel bei einem kurzfristigen Abmarsch von diversen tausend oder gar zehntausend Personen turbulente, nicht zuletzt operativ nachteilige Verhältnisse $z u$ befürchten waren. Ob die zusätzliche Aufgabe bei einem bewaffneten Konflikt von der Eisenbahn hätte bewältigt werden können, wenn die Truppentransporte höchste Priorität hatten ${ }^{44}$, muss dahingestellt bleiben, da die Entwicklung auf dem Kriegsschauplatz sowohl 1866 als auch 1870/71 ein Beanspruchen solcher Bahnleistungen erübrigte und folglich die dem Innenminister gegebene, offenbar unverbindliche Zusage nicht auf die Probe gestellt wurde.

\section{II.}

Die Unruhen in diversen deutschen Bundesstaaten im Gefolge der Julirevolution von 1830 und insbesondere Belgiens Kampf um Unabhängigkeit von den Niederlanden veranlassten Preußen zu einer Teilmobilmachung, die im Westen die Kriegsvorbereitung der Festungen einschloss; dazu gehörten auch die seit 1809 landesweit vorgesehenen Vorkehrungen zur Ausweisung von Bewohnern ${ }^{45}$. Obwohl von höheren Kommandobehörden alsbald verlangt ${ }^{46}$, konnte von einer raschen Bearbeitung und sachdienlichen Vorbereitung etwaiger Evakuierungen durch die Bezirksregierungen und die Landräte nicht die Rede sein, wie Mahnungen belegen, die zur zügigen Ermittlung der Auszuquartierenden und deren Aufnahmeorte drängten ${ }^{47}$. Das Zaudern der Zivilbehörden war nicht auf fehlenden Diensteifer zurückzuführen, sondern in erster Linie auf das Gebot heimlichen Vorgehens, um Aufsehen und Aufregung zu vermeiden ${ }^{48}$; das lässt sich unschwer

43 Ebd., Nr. 11311, S. 71f.

44 Siehe mit entsprechender Direktive aus dem Jahr 1866 Landesarchiv Nordrhein-Westfalen Abtlg. Westfalen, Münster (LAV NRW W), Oberpräsidium Nr. 602, Bd 4, fol. 81.

45 Siehe mit knappen Hinweisen Bernhard Sicken, Koblenz, Köln, Wesel. Militärisch-zivile Beziehungen in preußischen Festungsstädten 1815-1914. In: Preußens schwieriger Westen. Rheinisch-preußische Beziehungen, Konflikte und Wechselwirkungen. Hrsg. von Georg Mölich [u.a.], Duisburg 2003, S. $291 \mathrm{f}$.

46 LAV NRW R, BR 0009 Nr. 1191, fol. 2.

47 Ebd., fol 15.

48 Ebd., fol. $21 \mathrm{ff}$. 
anhand der mühsamen Sondierungen in Wesel belegen. Erst Ende Dezember 1832, nachdem ca. siebzehn Monate verstrichen waren, lag für diese Festungsstadt ein Ergebnis vor, das die Anzahl Auszuweisender auf 1070 Zivilpersonen (ca. 10\% der Städter) und auf 872 Militärpersonen (quotiert ungefähr 23 bis 24\%) bezifferte ${ }^{49}$. Die Unterbringung der zivilen Bewohner sollte in den rechtsrheinischen Kreisen Rees und Duisburg und der Militärangehörigen in den ebenfalls östlich des Stroms gelegenen, aber zwei, drei Dutzend Kilometer entfernteren Kreisen Düsseldorf und Solingen erfolgen, wobei die beiden Kreise außerdem 111 Soldatenfamilien (geschätzt ungefähr 30\% der Militärbevölkerung) aus der Festung Jülich beherbergen sollten und dennoch nicht als überbürdet galten, stand doch diesen Leuten weiterhin Servis und Brot zu. Ferner sollten 1400 zivile Einwohner (rund 48\% der Stadtbevölkerung) im Fall einer Bedrohung Jülich verlassen, für deren Unterkommen die linksrheinischen, stärker gefährdeten Kreise Grevenbroich, Gladbach, Neuß und Kempen vorgesehen waren. Zugleich war die Frage ihres Unterhalts angeschnitten ${ }^{50}$ und mit dem vagen Hinweis auf Selbstversorgung abgetan worden, sodass dem rheinischen Oberpräsidenten nur als Behelf blieb, bei unabweisbarem Bedarf Armenunterstützung durch die Bezirksregierungen und Kommunen trotz offensichtlich unzulänglicher Ressourcen $\mathrm{zu}$ empfehlen sowie auf private Almosen zu hoffen. Das Ungenügen dieser Mittel war nicht zu bezweifeln und wurde indirekt durch das vielfache Verlangen nach strikter Geheimhaltung der Planungen unterstrichen, die bei einer Verwirklichung viele Betroffene ins Elend gebracht hätten.

Die Anzahl der Auszuweisenden aus den Festungen im Westen, wie 1831/32 registriert, ist im Folgenden zusammengestellt, um den Umfang zu verdeutlichen. Für die divergierenden Ansätze bei der auszuquartierenden Militärbevölkerung findet sich keine Erklärung; de facto dürfte jeweils das Ermessen der Befehlshaber den Ausschlag gegeben haben.

49 Ebd., fol. 37 f. Siehe auch Vergleichende Zusammenstellung der Einwohnerzahl der Städte des Preußischen Staates von 1840-1855. In: Archiv für Landeskunde der Preußischen Monarchie, Bd 4, Berlin 1856, S. $244 \mathrm{f}$. Bei den Angaben für 1832 handelt es sich zum Teil um Schätzungen.

50 LAV NRW R, BR 0009, Nr. 1191, fol. $44 \mathrm{f}$. 
Tabelle 1: Anzahl der Auszuweisenden aus den Festungen im Westen

\begin{tabular}{lrrrrr}
\hline Stadt & Jahr & $\begin{array}{r}\text { Zivilpersonen } \\
\text { (absolut) }\end{array}$ & $\begin{array}{r}\text { Zivilpersonen } \\
\text { (in\%) }\end{array}$ & $\begin{array}{r}\text { Militärpersonen } \\
\text { (absolut) }\end{array}$ & $\begin{array}{r}\text { Militärpersonen } \\
\text { (in\%) }\end{array}$ \\
\hline Saarloluis $^{\mathrm{a}}$ & 1831 & 1360 & 32 & 446 & $14^{\mathrm{b}}$ \\
\hline Koblenz $^{\mathrm{c}}$ & 1831 & 3000 & 25 & 540 & 14 \\
\hline Köln $^{\mathrm{d}}$ & 1831 & $15000-18000$ & $22-27$ & 520 & 11 \\
\hline ülich $^{\mathrm{e}}$ & 1832 & 1400 & 48 & 400 & 30 \\
\hline Wesel $^{\mathrm{f}}$ & 1832 & 1070 & 9 & 872 & 24 \\
\hline Minden $^{\mathrm{g}}$ & 1831 & $?$ & $?$ & 616 & 29 \\
\hline
\end{tabular}

Bei allen Prozentangaben handelt es sich um Annäherungswerte

a LHA Ko, Best. 403, Nr. 1807, S. 109 f. Siehe auch Rudolf Kretschmer, Geschichte der Kreisstadt Saarlouis, Bd 4: Saarlouis 1680-1980, Saarlouis 1982, S. 942. Die folgenden, zum Teil lückenhaften Angaben zur Einwohnerschaft wurden relativierend anhand Vergleichende Zusammenstellung (wie Anm. 49), S. 242-246 ergänzt.

b Zu den Angaben über die Militärpersonen siehe GStA, PK I. HA Rep. 77 Ministerium des Innern, Tit. $332^{\text {bb }}$ Nr. 4 Bd 1, Düsseldorf, 14.10.1831

c LHA Ko, Best. 403, Nr. 11310, S. 27, Randbemerkung, sowie Nr. 1807, S. 140. Zusätzlich wurden für Ehrenbreitstein 600 Auszuweisende angegeben, siehe Belege wie vorstehend, sowie Böckling, Frauen (wie Anm. 3), S. 64. Lüdtke, »Gemeinwohl« (wie Anm. 2), S. 275, geht von 3500 aus Koblenz zu entfernenden Personen aus und hat vermutlich jene aus Ehrenbreitstein mitveranschlagt; Grundlage dieser Angaben waren Schätzungen; siehe LHA Ko, Best. 403, Nr. 1807, S. 141

d LHA Ko, Best. 403, Nr. 1807, S. 357. Bei Lüdtke, »Gemeinwohl« (wie Anm. 2), S. 276, findet sich - gestützt auf ein anderes Schriftstück - ein um ca. 20\% geringerer Ansatz, obwohl die Auszuquartierenden aus Deutz angeblich mitberücksichtigt sind; Beleg wie vorstehend S. 353. Die Akten sind demnach nicht widerspruchsfrei und lassen nur die Dimension erkennen.

e LAV NRW R, BR 0009 Nr. 1191, fol. $37 \mathrm{f}$. Ohne für die vorstehende Fragestellung relevante Erkenntnisse ist der Beitrag von Andreas Kupka, Der Armierungsplan der Festung Jülich von 1817. In: Festungsbaukunst in Europas Mitte. Festschrift zum 30-jährigen Bestehen der Deutschen Gesellschaft für Festungsforschung, Regensburg 2011 (= Festungsforschung, 3), S. 105-121.

f LAV NRW R, BR 0009 Nr. 1191, fol. $37 \mathrm{f}$.

g LAV NRW OWL, M 1 Pr, Nr. 139, fol. 15. Angaben über die bedürftigen Zivilbewohner fehlen, doch lassen die wiederholt lobend erwähnten Festungsausbauten mit einer Vielzahl Beschäftigter Rückschlüsse auf die Ortsarmut zu. Siehe LAV NRW OWL, M 1 Pr, Nr. 20, fol. 264, Nr. 294, fol. 30 f.

Das Krisenjahr 1840 mit seinen deutsch-französischen Spannungen führte vorübergehend zu Kriegsbesorgnissen. Ihren Niederschlag fanden die Vorkehrungen zum einen durch ein Abkommen zwischen der Wiener und der Berliner Regierung über die Führung der norddeutschen Bundestruppen, die aus operativ einsichtigen Gründen Preußen zugebilligt wurde, und zum anderen durch Schutzmaßnah- 
men vor allem im Rheinland, bei denen wiederum die Festungen im Mittelpunkt standen. Im Wesentlichen knüpften die Verantwortlichen an die Regelungen der Jahre 1831/32 an, wie die Vorschriften zum Ausquartieren Mittelloser bekunden ${ }^{51}$. Ergänzend kamen Anweisungen an die rheinischen und westfälischen Zivilbehörden für den Fall einer Invasion hinzu, die ältere Instruktionen präzisierten ${ }^{52}$. Das traf aber nicht für den beschwerlichen Unterhalt von Abgeschobenen zu, denn mehr als ein beiläufiger Passus, durch den in substanzarmen Formulierungen neuerlich auf die Pflicht zur Sorge für den Unterhalt Evakuierter hingewiesen wurde, fand sich in der Instruktion nicht.

Die österreichische Politik nach dem Scheitern der 48er-Revolution, die auf eine Restitution des Deutschen Bundes zielte und zum Fehlschlag der preußischen Unionspläne beitrug, führte in den Auseinandersetzungen über die Parteinahme im Verfassungskonflikt Kurhessens bis an die Schwelle des Kriegs, verbunden mit der Mobilisierung von Truppen und weiteren Vorkehrungen ${ }^{53}$. Hierzu rechneten in den besonders gefährdeten Gebieten, zu denen die preußischen Regionen in Ost- und Südwestfalen gehörten, sowohl Anweisungen zur Sicherung der Steuereinnahmen und -akten, die bei Gefahr nach Minden oder bei einem dortigen Risiko nach Wesel gebracht werden sollten ${ }^{54}$, als auch die Aufforderung an die (Bezirks-)Regierung Minden, administrativ die Aufnahme der aus der gleichnamigen Festung notfalls auszuweisenden Bewohner vorzubereiten ${ }^{55}$. Indessen mussten die Vorsichtsmaßnahmen nicht verwirklicht werden, da die Gefahren nach ein paar Wochen schwanden.

Vorwiegend beanspruchten nach der Jahrhundertmitte jedoch die rheinischen Festungen Aufmerksamkeit. Das traf u.a. für Wesel zu, obwohl dieses niederrheinische Bollwerk trotz grenznaher Lage weniger als die anderen festen Plätze gefährdet war und primär nur als Fluchtort für Staatskassen und höheres Behördenpersonal größere Wertschätzung erfuhr. In Wesel wurden im Jahr 1851 neuerlich die ärmeren Stadtbewohner erfasst, die sich nicht für längere Zeit verproviantieren konnten. Die Anzahl der insgeheim registrierten Zivilbewohner belief sich auf 2802 Städter (ca. 21\%) ) $^{56}$ demnach hatte sich die Schicht Bedürfti-

51 Ebd., fol. 63.

52 Ebd., fol. $64 \mathrm{ff}$.

53 Siehe zusammenfassend Jürgen Angelow, Von Wien nach Königgrätz. Die Sicherheitspolitik des Deutschen Bundes im europäischen Gleichgewicht (1815-1866), München 1996 (= Beiträge zur Militärgeschichte, 52), S. 154-160.

54 LAV NRW OWL, M 1 Pr, Nr. 139, fol. 26.

55 Ebd., fol. 29.

56 LAV NRW R, Br 0009, Nr. 1191, fol. 89f. Siehe zudem Vergleichende Zusammenstellung (wie Anm. 49), S. 244. 
ger im Vergleich zum Jahr 1832 mehr als verdoppelt. Der Zuwachs ist nicht in erster Linie demografisch zu erklären, sondern zum einen auf eine längere Verproviantierungsdauer zurückzuführen, die nun auf sechs Monate veranschlagt worden war und stärker als zuvor marginalisierend wirkte, und zum anderen der rigorosen Definition von Armut zuzuschreiben, die als Minimum für den Tageskonsum einer Familie fünfzehn Silbergroschen (1/2 Taler) ansetzte. Damit war formal die zur Ausquartierung qualifizierende Bedürftigkeit festgelegt, die andernorts in vergleichbarer Weise den Rahmen absteckte. Von einer solchen Analogie kann aber nicht bei der Verproviantierungsdauer von sechs Monaten die Rede sein, die bekanntlich seit ein, zwei Dezennien durchweg auf drei Monate reduziert worden war und mit dieser Befristung weiterhin den freilich unverbindlichen Maßstab bildete ${ }^{57}$.

Bestätigung fand diese zeitliche Begrenzung im Jahr 1859, als der Oberpräsident der Rheinprovinz, alarmiert durch die Teilmobilisierung der Armee im italienisch-französisch-österreichischen Krieg, eine Überprüfung der Anzahl Auszuweisender verlangte, die nicht zuletzt infolge überproportionalen Bevölkerungswachstums geboten erschien. In Köln rechnete man jetzt mit 40000 Zivilpersonen (34 bis 35\% der Einwohner), die notfalls die Stadt verlassen mussten, von denen der überwiegende Teil abschätzig als Almosenempfänger klassifiziert wurde ${ }^{58}$. Von ähnlichen Verhältnissen war in den anderen rheinischen Festungen auszugehen, wie sich in Wesel mit einer unterdessen auf 30 bis 31\% gestiegenen Quote bzw. 4337 Ortsansässigen zeigte, jedoch lag hier der Anteil der auf Armenunterstützung Angewiesenen aus strukturellen Gründen niedriger ${ }^{59}$. Mit einer auf 190 geschrumpften Anzahl rechneten in Wesel nur noch wenige Militärpersonen zu den Abzuschiebenden - für die anderen Städte fehlen die Anschläge -, doch ist nicht von einer grundlegenden materiellen Verbesserung auszugehen, ausschlaggebend war vielmehr eine revidierte Erfassung dieses Personenkreises, der inzwischen mehrheitlich den militärischen Status verloren hatte und der zivilen Seite zugerechnet wurde ${ }^{60}$, wodurch zugleich der drastische Anstieg Auszuquartierender eine Erklärung findet.

57 LAV NRW R, BR 0009 Nr. 1191, fol. $92 \mathrm{ff}$.

58 Siehe mit generellem Hinweis Pierre Ayçoberry, Köln zwischen Napoleon und Bismarck. Das Wachstum einer rheinischen Stadt, Köln 1996 (= Kölner Schriften zur Geschichte und Kultur, 20), S. 360 f., und Herres, Köln in preußischer Zeit (wie Anm. 17), S. 368 und S. 375.

59 LAV NRW R, BR 0009, Nr. 1191, fol. 99.

60 Siehe mit entsprechenden Erläuterungen Thomas Tippach, Koblenz als preußische Garnison und Festungsstadt. Wirtschaft, Infrastruktur und Städtebau, Köln, Weimar 2000 (= Städteforschung, Reihe A, 53), S. 11, Anm. 21. 
Neben der Aktualisierung des Informationsstandes war der rheinische Oberpräsident darauf bedacht, die Evakuierungen einzudämmen, da er bei rigorosem Vorgehen große Schwierigkeiten in der bevölkerungsreichen Region vorhersah ${ }^{61}$. Er drängte deshalb bei den lokalen Registrierungen sowie den aufsichtführenden Instanzen auf Mäßigung und plädierte indirekt für eine Nutzung des Ermessens. Zudem verlangte er festzustellen, wie viele Städter am Ort verbleiben könnten, falls ihnen eine begrenzte Selbstversorgung möglich sei und nur das Fehlende durch eine kommunale Beihilfe gedeckt werden müsse. Ferner sollte bedacht und geprüft werden, ob nicht auch für manchen ein Unterschlüpfen bei Verwandten in Betracht käme, um eine zusätzliche Reduzierung jener zu erzielen, die sonst Fürsorge durch die öffentliche Hand benötigten. Zur Drosselung der Abschiebungen trugen die Direktiven wenig bei, andererseits nahm man aber mit den Sondierungen in Kauf, dass die erwünschte Geheimhaltung nicht aufrechterhalten werden konnte und Misstrauen nicht nur bei den unteren Schichten aufkam, wenn amtliche Auskünfte über den Lebensunterhalt oder die Verwandtschaft eingeholt wurden. Es kann nicht verwundern, dass das Unbehagen über die militärische Ausweisungsbefugnis stieg, an deren Restriktion den überforderten Zivilbehörden so offenkundig lag, die hier zugleich als bürgerliche Sachwalter amtierten.

Von Festungsstädten konnten nach dem Kriegsleistungsgesetz von 1851 bei einer Bedrohung unentgeltliche Arbeiten unterschiedlicher Art durch das Militär verlangt werden ${ }^{62}$, soweit diese mit der Versetzung der Fortifikationsanlagen in den Kriegsstand »vorübergehenden Zwecken« dienten, wie der Gesetzgeber als Beschränkung festgelegt hatte. Eine genauere Spezifikation unterblieb ${ }^{63}$, die der wenige Jahre später beim ersten Anlass angerufene Kriegsminister unter Verweis auf die gebotene Geheimhaltung ausdrücklich ablehnte und somit die militärische Entscheidungsgewalt sicherstellte ${ }^{64}$. Also blieb der Umfang der Leistungen dem Gutdünken der lokalen Befehlshaber überlassen; das galt erst recht für Ausweisungen. An Streitigkeiten über das Abgrenzen der Arbeiten zu »vorübergehenden Zwecken« von solchen eines insgeheim dauerhaften Ausbaus der Anlagen mangelte es künftig nicht, bei denen die Städte von vornherein in einer ungüns-

61 LAV NRW R, BR 0009, Nr. 1191, fol. $92 \mathrm{ff}$.

62 Gesetz=Sammlung für die Königlichen Preußischen Staaten 1851, Berlin [1851], Nr. 20, S. 362 f. $\$ 3$.

63 Weder in der Begründung der Gesetzvorlage (»Allerhöchste Verordnung«) noch in der Diskussion des Entwurfs im Abgeordnetenhaus wurde diesem Passus nähere Beachtung zuteil. Siehe Stenographische Berichte über die Verhandlungen der beiden Häuser des Landtages. Haus der Abgeordneten 1850/51, Berlin 1851, hier Bd 2, S. 692-717, Bd 3, S. 155-158, Bd 4, S. 839-841. 64 GStA PK, I. HA, Rep. 77 Ministerium des Innern, Tit. 330 a, Nr. 32, fol. 1. 
tigen Position waren, weil ihnen ein Urteil über das fortifikatorisch Notwendige einschließlich der etwaigen Evakuierung von Einwohnern abgesprochen wurde.

\section{III.}

Ende März 1866 hatte Preußen wegen wachsender Spannungen mit Österreich Rüstungen eingeleitet ${ }^{65}$ und hiermit auf heimliche Kriegsvorbereitungen der Donaumonarchie reagiert. $\mathrm{Zu}$ den frühzeitigen Maßnahmen zählte die Armierung der Festungen Glatz, Neiße und Kosel im oberschlesischen Grenzgebiet sowie von Torgau und Wittenberg im preußisch-sächsischen Vorfeld ${ }^{66}$, ergänzt für die Zivilbehörden durch den vorsorglichen Hinweis auf die Befugnisse der Festungsbefehlshaber ${ }^{67}$, um bei einem eventuellen Einfall aus dem anrainenden Gebiet Österreichs und der vermutlichen habsburgischen Anhänger im Deutschen Bund ${ }^{68}$ gewappnet zu sein. Das diplomatische Ringen zwischen der Wiener und der Berliner Regierung um den Frieden, das von Kontroversen über eine Reform des Deutschen Bundes überlagert war, schlug fehl, da die Zusage wechselseitiger Demobilisierung uneingelöst blieb, als sich Österreich durch italienische Rüstungen herausgefordert sah. Folglich nahmen hüben und drüben die Kriegsvorbereitungen ihren Fortgang, wie die seit Anfang Mai schrittweise angeordnete Mobilmachung der preußischen Armee bekundet ${ }^{69}$. Die Phase erhöhter Kriegsbereitschaft mit ihrem Taktieren um eine gute Ausgangsposition ${ }^{70}$ endete Mitte Juni 1866, als Preußen den Deutschen Bund nach dessen mehrheitlicher Parteinahme (gewichtet) zugunsten Österreichs für erloschen erklärte und Truppen zur Verbes-

65 Siehe LAV NRW OWL, M 1 Pr, Nr. 312, fol. 45, sowie Angelow, Von Wien nach Königgrätz (wie Anm. 53), S. 242-249. Siehe dazu auch Heinz Helmert, Militärsystem und Streitkräfte im Deutschen Bund am Vorabend des preußisch-österreichischen Krieges von 1866, Berlin 1964 (= Militärhistorische Studien, N.F., 7), S. 201, der die ersten preußischen Rüstungen auf Anfang Januar 1866 datiert.

66 LAV NRW W, Oberpräsidium Nr. 534, Bd 1, fol. 4.

67 GStA PK, I. HA, Rep. 77 Ministerium des Innern, Tit. 330 a, Nr. 18, Bd 1, Berlin, 9.5.1866; siehe auch Archiwum Państwowe we Wrocławiu (APW), Akta miasta Kłodzka 5682, Glatz, 9.6.1866.

68 Siehe im Überblick Helmut Burckhardt, Deutschland, England, Frankreich. Die politischen Beziehungen Deutschlands zu den beiden westeuropäischen Großmächten 1864-1866, München 1970, S. 375-382.

69 Siehe Gordon A. Craig, Königgrätz, Wien, Hamburg 1966 (engl. Originaltitel: The Battle of Königgrätz, 1964), S. 63; Rudolf Lill, Beobachtungen zur preußisch-italienischen Allianz (1866). In: Quellen und Forschungen aus italienischen Archiven und Bibliotheken, 44 (1964), S. 468-485; Adam Wandruszka, Schicksalsjahr 1866, Graz [u.a.] 1966, S. 141 f.

70 Siehe detailreich zur Haltung der mindermächtigen Staaten Karl Lange, Bismarck und die norddeutschen Kleinstaaten im Jahre 1866, Berlin 1930, S. 93 ff. und S. 216. 
serung der Operationsbasis in die anrainenden Mittelstaaten Hannover und Kurhessen sowie in das Böhmen vorgelagerte Königreich Sachsen einrücken ließ. Außerdem waren die noch nicht armierten Festungen im Westen in Kriegsbereitschaft versetzt worden, und zwar vorzeitig Minden als exponierter Platz Anfang Mai $^{71}$ und Wesel, Köln(-Deutz), Koblenz und Saarlouis in den folgenden Wochen ${ }^{72}$.

Die fortifikatorische Vorbereitung nahm die Zivilbevölkerung nicht aus. Für deren Lebensbedarf fühlten sich bekanntlich weder die Militärs noch die Zivilbehörden verantwortlich - ausschlaggebend war neben prinzipiellen Gründen der offenkundige Geldmangel -, und folglich blieb die eventuell erforderliche Bevorratung wie üblich der individuellen Vorsorge überlassen. Dass eine mehrmonatige Verproviantierung viele Städter finanziell überforderte, war den staatlichen Repräsentanten klar, deshalb setzten sie weiterhin auf eine Ausweisung der ärmeren Schichten, zumal diese in einem anfänglich unpopulären $\mathrm{Krieg}^{73}$ als besonders anfällig für Unruhen galten. Auch von den Schwierigkeiten beim Erfassen dieser Personen und bei der Suche nach Aufnahmebezirken wussten die Behörden seit Langem $^{74}$, doch hatten weder die vielfältigen Bedenken noch die organisatorischen Probleme $\mathrm{zu}$ einer Modifikation des Ausquartierungsanspruchs geführt, geschweige denn zu dessen von den Militärs strikt abgelehnten Aufhebung. Als sich Ende Mai 1866 die Kriegsgefahr zuspitzte, sah sich die mittlerweile rund 118000 Einwohner ${ }^{75}$ zählende Stadt Köln deswegen neuerlich genötigt, das Evakuieren zahlreicher Bewohner vorzubereiten, deren Anzahl in einer ersten groben Schätzung auf 60000 beziffert ${ }^{76}$ und ein paar Wochen später zusammen mit jenen aus Deutz auf 80000 Personen veranschlagt wurde ${ }^{77}$, also tendenziell auf eine Entvölkerung hinauszulaufen schien. Ohne tatkräftige Unterstützung des Kölner

71 Diese Armierung hat Meinhardt, Festung Minden (wie Anm. 3) in seiner Darstellung über die Festung Minden übersehen.

72 LAV NRW R, BR 0009, Nr. 1764, fol. 88; LAV NRW W, Oberpräsidium Nr. 534, Bd 1, fol. 218 und fol. 232.

73 Frank Becker, Bilder von Krieg und Nation. Die Einigungskriege in der bürgerlichen Öffentlichkeit Deutschlands 1864-1913, München 2001 (= Ordnungssysteme. Studien zur Ideengeschichte der Neuzeit, 7), S. 135.

74 Siehe Lüdtke, »Gemeinwohl« (wie Anm. 2), S. 276, ferner Dieter Klein-Meynen, Anfangs eine gespannte Beziehung - Leben in der Festungs- und Garnisonsstadt Köln. In: Festungsstadt Köln. Das Bollwerk im Westen. Hrsg. von Henriette Meynen, Köln 2010, S. 392; Sicken, Koblenz, Köln, Wesel (wie Anm. 45), S. $291 \mathrm{f}$.

75 Siehe Ayçoberry, Köln zwischen Napoleon und Bismarck (wie Anm. 58), S. 412, Tab. 1.

76 LHA Ko, Best. 403, Nr. 11311, S. 11 f.

77 Ebd., S. 15f. Siehe in knapper Zusammenfassung auch Herres, Köln in preußischer Zeit (wie Anm. 17), S. 335, und zur ökonomischen Lage der unteren Bevölkerungsschichten S. 368 und S. 375, sowie ergänzend Ayçoberry, Köln zwischen Napoleon und Bismarck (wie Anm. 58), S. 194 und S. $360 f$. 
Regierungspräsidenten war eine derartige Aufgabe nicht einmal partiell zu lösen; das verdeutlichte die Bestimmung der Aufnahmegebiete, und bewog den skeptischen Leiter der Mittelbehörde dem Oberpräsidenten der Rheinprovinz einen zusätzlichen Rückgriff auf Quartiere im Regierungsbezirk Düsseldorf sowie überregional ausgreifend in Westfalen vorzuschlagen ${ }^{78}$. Eine erste Spezifikation der Kapazitäten zur Unterbringung der Abzuschiebenden folgte wenige Wochen später, die für den Kölner Distrikt bei Aussparung der festungsnahen Umgebung eine Quote von knapp einem Drittel dieser Städter vorsah, während der größere Teil in den Regierungsbezirken Düsseldorf, Aachen und notfalls Arnsberg Unterkunft finden sollte ${ }^{79}$. Die Planungen für die rheinische Metropole, die ohnehin durch ihre linksrheinische Lage begünstigt war, mussten nicht in die Tat umgesetzt werden, weil der Krieg den Westen nur vorübergehend bedrohte.

Im Vergleich zum Westen war die Lage im Osten weniger gefährdet, jedoch schloss die bei langfristiger Betrachtung günstigere Entwicklung militärtypische Wachsamkeit nicht aus, die beispielsweise infolge des polnischen Aufstands im Spätjahr 1830 geboten war und zu Kriegsvorbereitungen in der Festung Thorn veranlasste $^{80}$. Selbstverständlich kam es auch zu Vorsichtsmaßnahmen bei den Waffenplätzen im Landesinnern, insbesondere wenn sie im Vorfeld zu politisch distanzierten Bundesstaaten lagen ${ }^{81}$. Davon künden die Angaben über die Auszuweisenden aus Festungen in der Provinz Sachsen im Jahr $1864^{82}$, deren Erfassung vermutlich im Gefolge des Ringens um die Zukunft der Herzogtümer Schleswig und Holstein für die Behörden aktuell geworden war. Die Anzahl Abzuschiebender aus Magdeburg ${ }^{83}$ nach dem zugrunde gelegten Stand von 1859 war auf 21000 Personen (knapp 32\% der Einwohnerschaft) veranschlagt worden, aus Erfurt ${ }^{84}$ auf 7000

78 LHA Ko, Best. 403, Nr. 11311, S. $11 \mathrm{f}$.

79 Ebd., S. $15 f$.

80 GStA PK, I. HA, Rep. 77 Ministerium des Innern, Tit. 1303, Nr. 3, Marienwerder, 3.12.1830.

81 Siehe beispielsweise Uwe Niedersen, Heute noch vorhandene Elemente der Festung Torgau (ab 1810). Die Festung Torgau - sächsisch, später französisch kommandiert, schließlich preußisch übernommen. In: Kasernen - Lazarette - Magazine. Gebäude hinter den Wällen, Regensburg 2012 (= Festungsforschung, 4), S. 18.

82 Siehe GStA PK, I. HA, Rep. 77 Ministerium des Innern, Tit. 332 $2^{\text {bb }}$, Nr. 4, Bd 1, Kloster Roßleben, 24.8.1864, mit den Zahlenangaben für die vier erwähnten Städte.

83 Ende 1863 war die Modernisierung der Festung Magdeburg durch detachierte Forts beschlossen und 1866 der Ausbau forciert worden. Zum Ausbau siehe Bernhard Mai, Das befestigte Magdeburg. In: Magdeburg. Die Geschichte der Stadt 805-2005. Hrsg. von Matthias Puhle und Peter Petsch, Dössel (Saalkreis) 2005, S. 503f.

84 Erfurts Zukunft als Festung wurde schon Ende 1863 skeptisch beurteilt und endete dann 1873. Siehe Klaus-Dieter Kaiser, Erfurt als Garnison und Festung 1813-1873, Ebersdorf 2007, S. 152-155. Mit knappen Hinweisen zur Armierung siehe zudem Horst Moritz, Die Festung Petersberg unter Preußen: 1802-1918, Erfurt 2002, S. 53. 
Personen (annähernd 26\%, hier aber Stand von 1850), aus Torgau auf 3431 Personen (rund 48\%) und aus Wittenberg auf 600 Personen (knapp 6\%). Auffallend sind die beträchtlich divergierenden Quoten dieser Bewohner, die nur bedingt durch ein Wohlstandsgefälle zu erklären sind und eher auf unterschiedliche Maßstäbe bei der Bevorratung oder eine abweichende Dauer der Verproviantierung schließen lassen. Und das ist in der Tat für Torgau zu belegen, denn hier war man von einer Bevorratung für ein Jahr ausgegangen und damit von einer ungewöhnlich langen Zeit.

Wie erwähnt, waren die Festungen Wittenberg und Torgau sowie die schlesischen Pendants Glatz, Neiße und Kosel für den Schutz des Landes im Jahr 1866 von erheblicher Bedeutung, die deshalb frühzeitig armiert wurden ${ }^{85}$. Durch ihre Grenznähe waren die Waffenplätze Glatz, Neiße und Kosel exponiert, sodass sie zur Zuflucht für höhere Behörden und Kassen als ungeeignet galten ${ }^{86}$, wie der Oberpräsident der Provinz Schlesien frühzeitig den Berliner Ministerien bedeutete, der zugleich bemüht war, das Seine zur Unterstützung der Kampfkraft dieser Festungen beizutragen und die Voraussetzungen für eine Räumung von armen Unversorgten zu schaffen ${ }^{87}$. Es verdient in diesem Zusammenhang aber in erster Linie Beachtung, dass beinahe zur gleichen Zeit sein sächsischer Amtskollege höheren Orts für die Auszuquartierenden eingetreten war und die Bewilligung von Unterhaltsleistungen für die Arbeitsunfähigen sowie Maßnahmen zum Broterwerb für die Arbeitsfähigen angeregt hatte ${ }^{88}$, um durch staatliche Daseinsvorsorge $^{89}$ eine Notlage größeren Ausmaßes zu verhüten. Die Vorschläge zur sozialen Fürsorge durch die öffentliche Hand blieben ohne konkrete Auswirkungen, sie lassen jedoch die wachsende Verantwortlichkeit in der höheren Beamtenschaft bei offenkundiger Hilfsbedürftigkeit von Staatsbürgern erkennen.

Ist der Impuls positiv zu bewerten, weil er auf einen allmählichen Bewusstseinswandel hindeutet, so belastete zur gleichen Zeit konfessionspolitisches Mißtrauen anderenorts das Verhältnis von Bevölkerung und Staatsverwaltung. Die Loyalität schlesischer Katholiken und insbesondere von Geistlichen u.a. in Kosel wurde nämlich von einigen Behörden angezweifelt ${ }^{90}$; man unterstellte ihnen nicht

85 Siehe APW, Akta miasta Kłodzka 5681, Glatz, 1.4.1866, sowie mit punktuellem Hinweis Schlesische Provinzialblätter, Neue Folge 5, Breslau 1866, S. 382. Zur Versorgung der Besatzung mit Lebensmitteln, deren Vergütung zum Teil später strittig war, siehe GStA PK, I. HA, Rep. 77 Ministerium des Innern, Tit. 330 a , Nr. 23, Breslau, 15.7.1866, Breslau, 18.12.1866.

86 GStA PK, I. HA, Rep. 77 Ministerium des Innern, Tit. 332 ${ }^{\mathrm{bb}}$, Nr. 4, Bd 1, Breslau, 1.4.1866.

87 Ebd., Breslau, 11.5.1866 und Anlage.

88 Ebd., Roßleben, 24.8.1864.

89 Siehe auch Langewiesche, »Staat« und »Kommune« (wie Anm. 13), S. 630 und S. 633.

90 APW, Nadprezydium we Wrocławiu Nr. 349, fol. 19. 
nur Sympathie für Österreich, sondern auch die Unterstützung der Habsburgermonarchie. Angeblich hatte es neben einer den Gegner begünstigenden Agitation sogar vereinzelte Kirchenkollekten zu dessen Gunsten gegeben, wie lokal kolportiert und auf dem Amtsweg an den Minister für Geistliche, Unterrichts- und Medizinangelegenheiten berichtet worden war, der daraufhin zur scharfen Überwachung des Klerus in den katholischen Distrikten der Provinz aufforderte ${ }^{91}$. Die Anweisung trat einer schon Wochen zuvor geführten Beschwerde des Oberpräsidenten beim Breslauer Fürstbischof zur Seite, die aber flugs zurückgewiesen worden war und außerdem zu einem Ersuchen um Schutz der Geistlichkeit vor Verleumdungen geführt hatte ${ }^{92}$. Nach dem raschen Kriegsende war in der amtlichen Korrespondenz von solchen Animositäten nicht mehr die Rede, zumal die gerichtlichen Voruntersuchungen die Vorwürfe strafrechtlich nicht $\mathrm{zu}$ erhärten vermocht hatten und Anklagen offenbar unterblieben ${ }^{93}$.

Die Kriegsgefahr konkretisierte sich für die Bewohner der drei schlesischen Festungen Anfang April, als Armierungsarbeiten unter Rückgriff auf zivile Kräfte angeordnet wurden, die offenbar von beträchtlichem Ausmaß waren, wie für Glatz $\mathrm{zu}$ belegen ist ${ }^{94}$. Eine provisorische Erfassung der Einwohner, denen eine längere Verproviantierung nicht möglich war, folgte einige Wochen später und veranschlagte die Quote bei dreimonatiger Bevorratung auf rund 37 Prozent $^{95}$. Dieser Ansatz stellte sich indessen als überhöht heraus, nachdem zu Beginn des Monats Juni wegen wachsender Kriegsgefahr amtlich die Versorgung mit Lebensmitteln vorgeschrieben und eine anschließende Überprüfung der Vorräte angekündigt worden war ${ }^{96}$, denn nun sank die Quote Auszuquartierender auf gut 14\% (1336 Personen) ${ }^{97}$, die beim Bürgermeister freilich auf Skepsis stieß, der bei einer Nachkontrolle mit einem Anstieg rechnete; im Übrigen hielt er mehr als ein Drittel der Betroffenen für marschunfähig, deren Transport zu organisieren war.

Diese auf Glatz und wahrscheinlich weitere Kommunen zukommende Aufgabe scheint die (Bezirks-)Regierung Breslau als Aufsichtsorgan zu einem Hilfsangebot und mittelbar zur sozialen Fürsorge bewogen zu haben, in dem sie als staatlichen Vorschuß die Mittel für den Abmarsch der Ausgewiesenen und den

91 Ebd., fol. 38.

92 Ebd., fol. 36.

93 Ebd., passim.

94 Siehe APW, Akta miasta Kłodzka 5681, Glatz, 1.4.1866; Akta miasta Kłodzka 178, Protokollbuch der Stadtverordnetenversammlung, Nr. 1078, 1079, und Akta miasta Kłodzka 5682, Gl[atz], 21.4. [18]66.

95 APW, Akta miasta Kłodzka 5682, Randnotiz Gl[atz], 8.5.[18]66. Siehe zur Einwohnerschaft (Stand 1864) Schlesische Provinzialblätter (wie Anm. 85), S. 684.

96 APW, Akta miasta Kłodzka 5682, Glatz, 6.6.1866.

97 Ebd., Gl[atz], 19.6.[18]66. 
Abtransport der Marschunfähigen zusagte ${ }^{98}$ und hierdurch einem möglichen Desaster vorbeugte. Es war verständlich, dass bei den Aufwendungen auf strenge Sparsamkeit zu achten war, wobei als Norm die Sätze der Armenpflege gelten sollten. So durften beispielsweise die den unmittelbaren Bedarf übersteigenden Habseligkeiten nur auf eigene Kosten mitgenommen werden und waren daher de facto ausgeschlossen. Es war auch ungewiß und blieb späterer Klärung vorbehalten, ob den Evakuierten am Ort ihres vorübergehenden »Exils« eine Unterstützung aus öffentlichen Quellen zugebilligt werden konnte, denn offenbar hingen etwaige Beihilfen überwiegend vom Leistungsvermögen der aufnehmenden Kommune $a b^{99}$. Zur systematischen Vorbereitung gehörten ferner Absprachen mit einer privaten Eisenbahngesellschaft zum gestaffelten Abtransport dieser Leute ${ }^{100}$. Frühzeitig waren zudem die zur Aufnahme vorgesehenen Landkreise bestimmt worden ${ }^{101}$, von denen die meisten ca. 25 bis 40 km entfernt lagen. Die Repartition der Ausquartierten blieb den Landräten überlassen ${ }^{102}$ und ließ keine Überforderung erwarten, da auf keinen der Kreise mehr als 400 Personen entfielen ${ }^{103}$ und somit die befristet Unterzubringenden durchweg weniger als 3 Prozent der Kreisbevölkerung ausmachten. Glücklicherweise blieb der geplanten Evakuierung von Teilen der Glatzer Einwohnerschaft und wahrscheinlich auch jener der Festungen Neiße und Kosel die Bewährung erspart ${ }^{104}$. Zunächst hatten die zügigen Vorstöße preußischer Truppen Ende Juni im nordböhmischen Grenzgebiet ${ }^{105}$ von einem ungesäumten Vollzug der Ausweisungen Abstand nehmen lassen, und wenig später erübrigte sich das Vorhaben vollends, nachdem durch die Schlacht bei Königgrätz eine Vorentscheidung über den Kriegsausgang gefallen war ${ }^{106}$.

98 Ebd., Breslau, 1.6.1866.

99 Beim Unterhalt setzte man in erster Linie auf die pflichtgemäße Unterstützung durch die Gemeinden, daneben auf disponible Mittel der Armenfonds bei den (Bezirks-)Regierungen und auf private Wohltätigkeit. LHA Ko, Best. 403, Nr. 11311, S. 19. Zur Armenunterstützung der Kommunen siehe Gesetz=Sammlung für die Königlichen Preußischen Staaten 1843, Berlin [1843], Nr. 1, S. $12 \S 26$.

100 APW, Akta miasta Kłodzka 5682, Breslau, 21.6.1866.

101 Ebd., Gl[atz], 24.6.[18]67.

102 Ebd., Wohlau, 29.6.1866.

103 Ebd., Gl[atz], 24.6.[18]67.

104 Von einer tatsächlich vollzogenen Ausweisung in Glatz, Neiße und Kosel ging man hingegen im schlesischen Oberpräsidium aus, wie dem rheinischen Pendant im Frühjahr 1867 nach einem Auskunftsersuchen zur näheren Information mitgeteilt wurde. Siehe LHA Ko, Best. 403, Nr. 11311, S. 19. Für Glatz ist das nachweislich unzutreffend und für Neiße und Kosel bei analogen Verhältnissen zu vermuten, zumal in den punktuell ausgewerteten Quellen jeder Hinweis auf eine Abschiebung fehlt.

105 Craig, Königgrätz (wie Anm. 69), S. 97-126.

106 APW, Akta miasta Kłodzka 5682, Gl[atz], 24.6.[18]67. 
Offenkundig waren in Glatz die organisatorischen Vorkehrungen zum vorübergehenden Abschieben von Bewohnern weiter als andernorts gediehen, weil kurzfristig mit einem feindlichen Einfall gerechnet werden musste und deswegen die (Bezirks-)Regierung beizeiten Anordnungen getroffen hatte, die für den Magistrat zur Richtschnur wurden. Dennoch hatte der Bürgermeister mit mancherlei Schwierigkeiten bei einer tatsächlich vollzogenen Ausweisung gerechnet, so räumte er einige Monate später ein, fehlten doch der Kommune und den Staatsbehörden entsprechende Erfahrungen und überdies war das Verhalten widerwillig Ausquartierter nicht vorhersehbar. Dieses generelle Defizit bewog ein knappes Jahr später die Festungsstadt Erfurt zu einem Ersuchen um Auskunft über das Procedere einer Evakuierung und über die Glatzer Wahrnehmungen bei der vor Kurzem angeblich vollzogenen Abschiebung, um für künftige Fälle gerüstet zu $\operatorname{sein}^{107}$, da es landesweit an verbindlichen Bestimmungen und zeitgemäßen Regelungen mangelte.

Das Ermitteln der Abzuschiebenden und der Ausweichquartiere musste bekanntlich ohne Aufsehen erfolgen, um Unruhe unter den vermutlich Betroffenen $\mathrm{zu}$ vermeiden. Ohne genauere Kenntnisse war indessen weder ein sorgfältiges Erfassen bedürftiger Städter noch ein Abschätzen der Kapazitäten zu deren Unterbringung möglich, sodass eine Armierung durchweg zu heimlichen, denunziatorisch anmutenden Erkundigungen zwang, um auf tragfähiger Grundlage die erforderlichen Vorbereitungen treffen zu können. Die überwiegend den Kommunen überlassenen Sondierungen spiegeln sowohl das Bemühen um Diskretion als auch die insgeheimen Vorbehalte mancher Amtsinhaber, die ihre zögernde Mitarbeit mit teils zutreffenden, teils vorgeschobenen Argumenten zu rechtfertigen und Mahnungen flugs zu parieren wussten. Von diversen Nachfragen vorgesetzter Dienststellen abgesehen, unter denen an erster Stelle die Oberpräsidenten wegen ihrer koordinierenden Aufgaben $\mathrm{zu}$ nennen sind ${ }^{108}$, hat diese Problematik anscheinend keinen breiteren Niederschlag in den Akten gefunden, wenn man vom zufällig überlieferten Bericht eines Kölner Regierungsrats absieht ${ }^{109}$, der sich bei der Suche nach Unterkünften über ängstliche, offensichtlich verantwortungsscheue Bürgermeister und unkooperative desinteressierte Landräte beschwerte, sodass er sich schließlich zur Erfüllung seines Auftrags genötigt sah, anhand der Listen der Gebäudesteuer den belegbaren Quartierraum festzulegen und somit zu einem Indikator Zuflucht zu nehmen, der nicht mehr als eine grobe Hilfe bot, aber schwerlich Ausgewogenheit gewährleistete.

107 Ebd., Erfurt, 15.4.1867.

108 Siehe dazu generell LAV NRW OWL, M 1 Pr, Nr. 139, fol. 68, sowie mit Beispielen LAV NRW R, BR 0009, Nr. 1191, fol. 2, 92 ff., ferner LHA Ko, Best. 403, Nr. 11311, S. 29 f. und S. 65 f.

109 LHA Ko, Best. 403, Nr. 11311, S. $45 \mathrm{ff}$. 
Unauffällige Planungen zur Ausweisung waren kennzeichnend, allerdings kam es manchmal auch zu einem transparenten Vorgehen, wie für Torgau zu belegen ist ${ }^{110}$. Der Magistrat hatte angesichts wachsender Kriegsgefahr Ende Mai 1866 die Bevölkerung durch eine öffentliche Bekanntmachung zur dreimonatigen Verproviantierung binnen eineinhalb Wochen aufgefordert - zwei Jahre zuvor waren merkwürdigerweise zwölf Monate angesetzt worden -, ferner über die anschließende Kontrolle der Bevorratung informiert und jenen für den Fall einer Einschließung das Ausquartieren angekündigt, denen eine hinlängliche Bevorratung verwehrt war. Überdies unterrichtete er ein paar Wochen später über Art und Menge der Lebensmittel, die nach militärischer Erfahrung üblicherweise als Bedarf angesetzt wurden ${ }^{111}$, und legte somit nachträglich Richtlinien vor, die auf Unsicherheiten beim Überprüfen der eingelagerten Versorgungsgüter schließen lassen. Ob diese Empfehlungen noch umgesetzt wurden, ist zweifelhaft, denn wenig später war die kriegsentscheidende Wende eingetreten, die das Verproviantieren erübrigte.

Wie bereits erwähnt, kam der Sicherung der Kassenbestände und -bücher bei einem Einfall hohe Bedeutung zu, die deshalb den Dienststellen wiederholt in Erinnerung gerufen wurde ${ }^{112}$. Außerdem waren die Festungen als Rückzugsorte für die (Bezirks-)Regierungen vorgesehen, wenn das ihrer Verantwortung unterstehende Gebiet besetzt zu werden drohte, um dem Land die Kompetenz der höheren Beamtenschaft zu erhalten, dem Gegner den Zugriff auf die Ressourcen $\mathrm{zu}$ erschweren ${ }^{113}$ und später eine rasche Wiederaufnahme der Verwaltung $\mathrm{zu}$ gewährleisten. Einen vorzeitigen Abzug suchte das Staatsministerium freilich zu unterbinden, das deswegen im Mai 1866 in einem Rundschreiben die leitenden Behörden notfalls zum Ausweichen in noch unbesetzte Teile ihres Distrikts er-

110 Siehe Horst Müller, Die sächsisch-preußische Festung Torgau (1810-1893), Torgau 1995 (= Kleine Schriften des Torgauer Geschichtsvereins, 5), S. 65f.

111 Eine vergleichbare Aufstellung war Ende April 1866 vom lokalen Festungskommandanten dem Glatzer Bürgermeister als Leitlinie an die Hand gegeben worden. APW, Akta miasta Kłodzka 5682, Glatz, 27.4.1866.

112 Siehe LAV NRW R, BR 0009, Nr. 1191, fol. 18, und ferner mit einer Anweisung für die Zivilbehörden in den westlichen Provinzen, fol. 64ff., sowie ergänzt und modifiziert LAV NRW OWL, M 1 Pr, Nr. 139, fol. 69ff. Zur Suche nach einem sicheren Verwahrungsort für die Kassenbestände z.B. in Wesel siehe LAV NRW R, BR 0009, Nr. 1911, fol. 73f. Für die Berliner Staatskassen war Stettin als Fluchtort vorgesehen; GStA PK, I. HA, Rep. 77 Ministerium des Innern, Tit. 332 ${ }^{\text {bb }}$, Nr. 4, Bd 2, Berlin, 27.6.1866.

113 Wie für die Provinz Sachsen zu belegen ist und anscheinend generell maßgebend war, sollte bei einer Invasion die Erhebung staatlicher Steuern ausgesetzt werden, jedoch unter Aufrechterhaltung der Ansprüche. GStA PK, I. HA, Rep. 77 Ministerium des Innern, Tit. 332 ${ }^{\mathrm{bb}}$, Nr. 4, Bd 2, Magdeburg, 9.5.1866. 
munterte ${ }^{114}$, da der Staatsregierung an einer ordnungsgemäßen Amtsführung so lange wie möglich und demnach der sinnfälligen Handhabung der Landesherrschaft gelegen war. Vom großen Interesse an einer kontinuierlichen Verwaltung in krisenhafter Lage kündet zudem, dass künftig auch die Landräte in ihren Kreisen ausharren und ihren Pflichten nachkommen sollten. Denn als Beamte und zugleich durch das Vertrauen der Kreisangehörigen gewählte Amtsinhaber schien ihr Verbleiben ratsam zu sein ${ }^{115}$, um der Willkür etwaiger Besatzer entgegenzutreten.

Das Abschieben mittelloser Einwohner stieß Mitte der 1860er Jahre bei den Behörden zunehmend auf Kritik, galt doch bei größeren Festungsstädten ein Vollzug bei einer auf Tausende und Abertausende angestiegenen Menge Ausgegrenzter als problematisch, wenn nicht gar unausführbar, sodass schließlich einige Oberpräsidenten auf eine für das Militär akzeptable Modifikation bedacht waren, die nicht auf einen generellen Verzicht, sondern auf eine merkliche Einschränkung der Evakuierungen zielte ${ }^{116}$. Die daraufhin im Frühsommer 1866 durch das Staatsministerium eingehend erörterte Materie führte allerdings nicht zu einer Neuregelung, weil die unbehinderte Behauptung dieser Bollwerke für das Kriegsressort absolute Priorität hatte, während die Härte einer zwangsweisen Ausquartierung zwar bedauert, jedoch im Kriegsfall als unvermeidbar bezeichnet wurde ${ }^{117}$. Etwaige Ausweisungen sollten aber fortan je nach den Umständen erfolgen, und somit gab es nun eine gewisse Handhabe $\mathrm{zu}$ einem moderaten Vorgehen. Trotzdem blieben die Bedenken der zivilen Amtsinhaber bestehen, von denen mittelbar die Belehrung einer skeptischen Mittelbehörde durch den rheinischen Oberpräsidenten kündet, als sich dieser im Jahr 1867 mit zwiespältigen Argumenten zur Verteidigung von Evakuierungsplänen gezwungen sah ${ }^{118}$.

Mit den Aussichten auf ein maßvolles Vorgehen war die Pflicht zur Vorsorge nicht aufgehoben. Daher fanden die behördeninternen Diskussionen über die Evakuierungsdistrikte nach dem Krieg ihre Fortsetzung, zumal die eiligen Sondierungen während der Armierungen keine zufriedenstellende Klärung gebracht hatten. Die Initiative zu einer angemessenen Regelung, die offenbar durch Frankreichs Absichten zum Erwerb Luxemburgs und die daraus resultierenden Spannungen zusätzliche Aktualität erhielt, ging im Frühjahr 1867 vom Oberpräsidenten der vor

114 LAV NRW OWL, M 1 PR, Nr. 139, fol. 49, LAV NRW R, BR 0009, Nr. 1191, fol. 103.

115 GStA PK, I. HA, Rep. 77 Ministerium des Innern, Tit. 332 ${ }^{\mathrm{bb}}$, Nr. 4, Bd 1, Breslau, 17.5.1866, Liegnitz, 18.5.1866; vgl. auch ebd., Breslau, 2.12.1867.

116 LAV NRW OWL, M 1 Pr, Nr. 139, fol. 48, LHA Ko, Best. 403, Nr. 5373, S. 73.

117 Ebd. und ferner GStA PK, I. HA, Rep. 77 Ministerium des Innern, Tit. 332 ${ }^{\text {bb }}$, Nr. 4, Bd 1, Berlin, 16.5.1866.

118 LAV NRW R, BR 0009, Nr. 1191, fol. 134. 
allem gefährdeten Rheinprovinz aus ${ }^{119}$ und lief in erster Linie auf Vorkehrungen für Ausquartierungen aus Köln und Deutz hinaus. Hier stellte sich die Anzahl Betroffener nach vorsichtigen Schätzungen auf ungefähr 80000 Personen, von denen bekanntlich nur eine Minderheit im Regierungsbezirk Köln untergebracht werden konnte, sodass auf anrainende Gebiete zurückgegriffen werden musste. Hauptsächlich richtete sich das Interesse auf den Düsseldorfer Regierungsbezirk, ohne dort aber auf Entgegenkommen zu stoßen, wie ausweichende Antworten des Regierungspräsidenten und seine Hinweise auf die zahlreiche Fabrikarbeiterschaft, den beträchtlichen Wohnungsmangel und die erforderliche Lebensmittelzufuhr bekunden; als Ausweg schlug er deswegen kurzerhand das Belegen westfälischer Orte und demnach ein Ausgreifen auf die Nachbarprovinz vor ${ }^{120}$. Nur zögernd wurde schließlich eine Kapazität von 7700 Plätzen zur Aufnahme der Kölner und Deutzer ausfindig gemacht ${ }^{121}$ und somit eine erstaunlich kleine Menge für den zunehmend industrialisierten Düsseldorfer Regierungsbezirk ${ }^{122}$. Beachtung verdient, dass erstmals ohne Umschweife die vom raschen Wandel geprägten sozioökonomischen Strukturen ins Feld geführt wurden, die einem Abschieben Mittelloser entgegenstanden und eine Aufnahme andernorts erschwerten. Zur gleichen Zeit war der Oberpräsident der Provinz Sachsen ebenfalls abschlägig beschieden worden, der für einen Teil der zu evakuierenden Magdeburger die Nachbarprovinz Brandenburg zur Aufnahme vorgeschlagen hatte ${ }^{123}$, weil angeblich die nächstgelegenen, zur Unterbringung vorgesehenen Landgebiete Brandenburgs als allzu dürftig und folglich ungeeignet galten und andere, bessergestellte Distrikte als Reserve für etwaige Ausquartierungen aus Spandau vorgehalten werden sollten. Hüben wie drüben war den Einwänden eine gewisse Plausibilität nicht abzusprechen, eine Lösung der Problematik für größere Festungsstädte wurde ersichtlich schwieriger.

119 LHA Ko, Best. 403, Nr. 11311, S. $21 \mathrm{ff}$.

120 Ebd., S. $25 \mathrm{ff}$. und S. $35 \mathrm{f}$.

121 Ebd., S. 35f. Siehe mit knappen Hinweisen auch Herres, Köln in preußischer Zeit (wie Anm. 17), S. 335.

122 Zum Bevölkerungswachstum im Regierungsbezirk Düsseldorf siehe Verwaltungsgrenzen in der Bundesrepublik Deutschland seit Beginn des 19. Jahrhunderts, Hannover 1977 (= Veröffentlichungen der Akademie für Raumforschung und Landesplanung. Forschungs- und Sitzungsberichte, 110), S. 202f. und S. 206-208.

123 GStA PK, I. HA, Rep. 77 Ministerium des Inneren, Tit. 332 ${ }^{\text {bb }}$, Nr. 4, Bd 1, Potsdam, 12.5.1866. 


\section{IV.}

Nach dem Beilegen der Krise über den Besitz Luxemburgs ruhten die Bemühungen um Quartierorte, die den Zivilbehörden bei wachsenden Zweifeln an der Zweckmäßigkeit der Abschiebungen durchweg Verdruss bereiteten. Mit dem Ausbruch des Deutsch-Französischen Krieges, dem wenige Tage zuvor der Befehl zur Festungsarmierung in den westlichen Provinzen vorangegangen war ${ }^{124}$, änderte sich schlagartig die Lage, denn eine Invasion des kriegserfahrenen Gegners musste ins operative Kalkül einbezogen werden. Die Menge der Auszuweisenden, deren Aufnahmeorte nach Meinung des betont optimistischen Innenministers längst bestimmt sein sollten ${ }^{125}$, stellte sich nach überschlägigen Berechnungen für Köln-Deutz nunmehr auf 100000 Personen $^{126}$ (ungefähr 75\% der Einwohner), war also binnen Kurzem kräftig gestiegen, und selbst für Koblenz-Ehrenbreitstein waren 12000 Personen (46\%) in Anschlag gebracht, deren beträchtliche Verminderung jedoch als möglich galt ${ }^{127}$, während auf Wesel nur 2980 Personen $^{128}$ (ca. 21\% der Ortsansässigen) und auf Saarlouis $1200^{129}$ (geschätzt 26-27\%) entfielen. Für die unterschiedlichen Ansätze, die zum Teil von denen früherer Jahre beträchtlich abwichen, findet sich keine Erklärung; möglicherweise waren die

124 LHA Ko, Best. 403, Nr. 11311, S. 71f. Die »starke Rheinfront« mit ihren Festungen bildete in den Plänen des Generalstabschefs Moltke zur offensiven Kriegführung einen wichtigen Rückhalt. Siehe Eberhard Kaulbach, Der Feldzug 1870 bis zum Fall von Sedan. Zur deutschen militärischen Führung in heutiger Sicht. In: Entscheidung 1870. Der Deutsch-Französische Krieg. Hrsg. vom MGFA durch Wolfgang von Groote und Ursula von Gersdorff, Stuttgart 1970, S. 48. Zum operativen Vorteil der raschen Mobilisierbarkeit des preußischen Heeres siehe Wilfried Radewahn, Europäische Fragen und Konfliktzonen im Kalkül der französischen Außenpolitik vor dem Krieg von 1870. In: Europa vor dem Krieg von 1870. Mächtekonstellation - Konfliktfelder - Kriegsausbruch. Hrsg. von Eberhard Kolb, München 1987 (= Schriften des Historischen Kollegs, Kolloquien, 10), S. $58 \mathrm{f}$.

125 LHA Ko, Best. 403, Nr. 11311, S. 71 f.

126 Ebd., S. 41 ff. und S. 80 ff. Vgl. Herres, Köln in preußischer Zeit (wie Anm. 17), S. 368, der Mitte des Jahrhunderts rund zwei Drittel der erwerbstätigen Kölner zur Unterschicht zählt.

127 LHA Ko, Best. 403, Nr. 11311, S. 101ff.; siehe zudem Böckling, Frauen (wie Anm. 3), S. 64, und Manfred Böckling, Als Wallhausen Preußens Armee mobilmachte. Eine Panne an der Nahe im Jahr 1882 und die Mobilmachungs-Vorbereitungen des VIII. preußischen Armeekorps in der Rheinprovinz. In: Jahrbuch für westdeutsche Landesgeschichte, 35 (2009), S. 548-550, sowie zur Einwohnerschaft von Koblenz und Ehrenbreitstein Tippach, Koblenz als preußische Garnison (wie Anm. 60), S. 39.

128 LHA Ko, Best. 403, Nr. 11311, S. 99f. Der Ansatz auszuweisender Weseler lag zunächst bei 5000 und hätte somit rund 35\% der Einwohner betroffen. Siehe LHA Ko, Best. 403, Nr. 11311, S. $65 \mathrm{f}$

129 Ebd., S. 61ff. 
Differenzen auf divergierende Maßstäbe beim Festlegen von Bedürftigkeit zurückzuführen, möglicherweise aber auch auf die Absicht zur Demonstration der Unausführbarkeit allzu großer Ausweisungen, wie sich für Köln-Deutz mit der exorbitant hohen Quote von 75 Prozent bei vermutlichem Einbeziehen von Teilen der unteren Mittelschicht aufdrängt und außerdem durch die kaum zu bewältigende Suche nach Unterkünften in der Region unterstrichen wird.

Diese Aufgabe wurde in der Tat zur nicht zu meisternden Herausforderung. Darauf deutet zum einen hin, dass neben den Regierungsbezirken Köln und Düsseldorf - letzterer wollte im Unterschied zu 1866 jetzt 13000 Plätze verfügbar machen ${ }^{130}$ - auch Unterkunftskapazitäten in den Provinzen Westfalen und Hessen-Nassau beansprucht werden sollten ${ }^{131}$, und das zeigt zum anderen, dass der Innenminister mit Zustimmung des Staatsministeriums angesichts der unbestreitbaren Problematik der Evakuierungen und ihrer Folgen mit Nachdruck für ein restriktives Vorgehen plädierte ${ }^{132}$ und Abschiebungen auf den Fall einer konkreten Gefahr beschränkt wissen wollte. Damit nicht genug: Die gleichzeitige überraschende Zusage des Kriegsministers, den Abzug mittelloser Städter durch einen kostenlosen militärischen Bahntransport zu erleichtern ${ }^{133}$, verweist auf eine sich wandelnde Einschätzung des erheblichen Bedenken ausgesetzten Ausweisungsanspruchs und ließ vermehrt nach Lösungen suchen, um ein Chaos bei einer massenhaften Ausquartierung im Festungsumland zu verhindern.

Die für Köln-Deutz projektierten Evakuierungen hielt selbst der durch die verbreitete Skepsis ermutigte Innenminister für unrealistisch ${ }^{134}$, denn neben den zu erwartenden Schwierigkeiten bei der Organisation und beim Transport galt die Versorgung als ungeklärt, die voraussichtlich zu einem nicht geringen Teil dem Staat zufallen und beträchtliche Aufwendungen verursachen würde, weil sonst Unruhen, wenn nicht Revolten zu befürchten waren. Die Befugnis war nach Ansicht des Ministers im bisherigen Modus nicht aufrechtzuerhalten, der deswegen die Materie zur baldigen Beratung im Staatsministerium empfahl und trotz der noch im Jahr 1866 erfolgten Ablehnung auf eine Modifikation drängte. Sein Vorstoß hatte Erfolg, erklärte doch nunmehr Ministerpräsident von Bismarck die Vorschrift von 1809 für veraltet und nur noch auf kleinere Festungen für anwendbar $^{135}$. Der Ansicht stimmte der durch die Waffenerfolge von 1864 und 1866

130 Ebd., S. 89f.

131 Ebd., S. $80 \mathrm{ff}$.

132 Ebd., S. $95 \mathrm{ff}$.

133 Ebd., S. 71f.

134 GStA PK, I. HA, Rep. 77 Ministerium des Innern, Tit. 330a, Nr. 18, Bd 1, Berlin, 29.7.1870.

135 Ebd., verhandelt Berlin, 30.7.1870. Siehe auch resümierend Die Protokolle des Preußischen Staatsministeriums 1817-1934/38, Bd 82, fol. 109 (Mikrofiches-Ausgabe). 
politisch gestärkte Kriegsminister zu; mehr noch, er fügte unverblümt an, die Regelung stamme aus »einer Zeit der Omnipotenz des Staates «136, der sich kurzerhand über die Folgen einer Ausweisung hinweggesetzt habe, während nach jetzigem Verständnis der öffentlichen Hand bei einer Notlage die Verpflichtung zur Daseinsvorsorge obliege. Für Festungsstädte wie Köln, Koblenz, Magdeburg etc. komme deshalb eine Abschiebung nicht mehr in Betracht, so räumte der Chef des Kriegsressorts realistisch ein, zumal bedeutende Waffenplätze bei einer Einschließung nicht von jeglicher Zufuhr abgeschnitten werden könnten und zumindest eine begrenzte Versorgung zu unterstellen sei. Nicht genug damit, er schätzte derzeit eine größere Gefährdung Kölns als unwahrscheinlich ein und riet von Vorbereitungen zum Ausquartieren aus der Stadt ab. Tatsächlich bestätigten die Grenzschlachten die Ansicht des Kriegsministers, der daraufhin offiziell dem Kölner Festungsgouverneur den Verzicht auf alle Maßnahmen zu Lasten der Bevölkerung nahelegte und den zivilen Bedenken Rechnung trug ${ }^{137}$.

Nicht nur die festen Plätze im Rheinland wurden 1870 armiert, sondern u.a. auch die ostwestfälische Festung Minden, obwohl hier von einer grenznahen Lage nicht auszugehen war und die Nachbarschaft mit Argwohn beobachteter Bundesstaaten im Jahr 1866 ihr Ende gefunden hatte. Die vom westfälischen Oberpräsidenten wenige Tage nach der Kriegserklärung Frankreichs verlangte Auskunft über die eventuell Auszuquartierenden fand eine rasche, im Vergleich zu den Angaben andernorts differenzierte Antwort durch den Oberbürgermeister ${ }^{138}$ und kategorisierte die Mindener Stadtbevölkerung folgendermaBen $^{139}$ :

136 GStA PK, I. HA, Rep. 77 Ministerium des Innern, Tit. 330a , Nr. 18, Bd 1, verhandelt Berlin, 30.7.1870.

137 Ebd., Berlin, 23.8.1870. Ob hiermit auch auf eine kritische Stimmung in der katholischen Bevölkerung Rücksicht genommen wurde, muss dahingestellt bleiben. Siehe mit einem Hinweis zur »Mobilisation gegen den inneren Feind « Alexander Seyferth, Die Heimatfront 1870/71. Wirtschaft und Gesellschaft im deutsch-französischen Krieg, Paderborn [u.a.] 2007 (= Krieg in der Geschichte, 35), S. 300.

138 LAV NRW OWL, M 1 Pr, Nr. 139, fol. 109f. und fol. $122 \mathrm{ff}$.

139 Ebd., fol. 123. Siehe auch Meinhardt, Festung Minden (wie Anm. 3), S. 112-114. 
Tabelle 2: Mindener Stadtbevölkerung

\begin{tabular}{lrr}
\hline Auszuquartierende & absolut & in\% \\
\hline Freiwillig Wegziehende & 1000 & 8 \\
\hline Ortsfremde Auszuweisende & 1000 & 8 \\
\hline Uneingeschränkte Selbstversorger & 2685 & 21 \\
\hline Befristete Selbstversorger (sechs bis acht Wochen) & 2875 & 23 \\
\hline Militärisch Benötigte (Helfer) & 125 & 1 \\
\hline Auszuweisende wegen Proviantmangels & 4876 & 39 \\
\hline Summe ortsanwesender Zivilpersonen & 12501 & 100 \\
\hline
\end{tabular}

Zugleich bat das mit der zeitgenössischen Kritik am Ausweisungsanspruch offenbar vertraute Stadtoberhaupt, nur im Notfall Evakuierungen vorzunehmen und dann ungesäumt Sonderzüge zum Transport bereitzustellen. Die fundierte Auflistung mit vermutlich repräsentativen Zügen für nicht industrialisierte kleinere Städte wurde für die Repartition der Abzuschiebenden auf die Umlandgemeinden maßgebend, die unter tatkräftigem Mitwirken des Oberpräsidenten rasch vorgenommen war und sowohl Kreise in den Regierungsbezirken Minden und Münster als auch ungescheut Städte und Ämter des Landdrosteibezirks Osnabrück der neuen Provinz Hannover einbezog ${ }^{140}$. Zu Widersprüchen in den Aufnahmeorten kam es anscheinend nicht, jedoch gab es noch nachträglichen Klärungsbedarf über den zulässigen Umfang der mitzunehmenden Habseligkeiten und den Schutz zurückgelassenen Eigentums; vorsorgliche Regelungen bei geringem Aufwand für die öffentliche Hand ließen nicht lange auf sich warten.

Der Schwerpunkt des Kriegsgeschehens lag 1870/71 unbestreitbar im Westen, allerdings waren andere Gebiete wie die küstennahen Regionen an der Ostsee ebenfalls bedroht ${ }^{141}$. Das traf neben dem kurzfristig von der französischen Kriegs-

140 Siehe zum Folgenden LAV NRW OWL, M 1 Pr, Nr. 139, fol. 131f. Detaillierte Listen zur Repartition finden sich auch ebd., fol. $144 \mathrm{f}$.

$141 \mathrm{Zu}$ den Operationen französischer Seestreitkräfte in der Ostsee siehe Hans-Justus Kreker, Die französische Marine im Kriege von 1870/71. In: Marine-Rundschau, Zeitschrift für Seewesen, 70 (1973), S. 278-282, sowie in knapper chronologischer Übersicht mit dem Hinweis auf die Bedrohung der Festung Kolberg Albert Röhr, Deutsche Marinechronik, Oldenburg, Hamburg 1974, S. 70. Vgl. mit Bemerkungen zum Küstenschutz auch Seyferth, Die Heimatfront (wie Anm. 137), S. 245 und S. 288-291. 
marine blockierten Kriegshafen Kiel ${ }^{142}$ für die Städte Stralsund, Swinemünde, Kolberg und Pillau $\mathrm{zu}^{143}$, nachdem die gegnerischen Panzerschiffe im August 1870 ihren Vorstoß nach Osten ausgeweitet hatten, ohne auf große Gegenwehr zu stoßen. In der Stadt Pillau, die mit der angrenzenden Festung den Seeweg durch das Frische Haff nach Königsberg deckte, hatte man angesichts der Gefahr flugs ein Schiff auf Bereithaltung gechartert ${ }^{144}$, das den Abtransport von 700 Städtern im Fall eines Angriffs übernehmen sollte, weil man mit größeren Eisenbahnkapazitäten nicht rechnen konnte. Die Bootscharter erwies sich indessen als unnötig, denn zu einer tatsächlichen Gefährdung kam es weder hier noch andernorts. Anschließend folgte in hergebrachter Weise eine längere Diskussion über die Kostenübernahme, für die der federführende Oberpräsident der Provinz Preußen (1878 geteilt in Ostpreußen und Westpreußen) staatliche Mittel beanspruchte, die der Innenminister erst nach längerem Hin und Her bewilligte ${ }^{145}$.

\section{V.}

Die 1870 erfolgte Verständigung im Staatsministerium über eine Beschränkung des Ausweisungsanspruchs hatte als interministerielle Regelung zwar praktische Bedeutung, doch hob sie die den Befehlshabern zugebilligten Befugnisse nicht auf und schmälerte keineswegs deren prinzipielle Verantwortlichkeit für den Kampfwert der Festung. Dennoch kam es bei manchen Kommandanten zur Verunsicherung über das Verhältnis zur Zivilbevölkerung im Gefahrenfall. Davon künden Anfragen des Kölner Festungsgouvernements u.a. im Spätjahr 1882 zur Lebensmittelversorgung ${ }^{146}$, die vom Kriegsministerium aber nur zögernd und ausweichend beschieden wurden, da das Ressort eine grundsätzliche, wahrscheinlich kontroverse Erörterung unter den Regierungsmitgliedern und erst recht eine etwaige Thematisierung in der Öffentlichkeit vermeiden wollte und sich mit dem Status quo zu arrangieren wusste. Immerhin war der Kriegsminister nun zur begrenzten Beteiligung an einer vorsorglichen Verproviantierung zugunsten der Zivilbewohner bereit; insgeheim hatte er nämlich die Militärverwaltung zur Anlage von Verpflegungsvorräten für bis zu 10 Prozent der Einwohner angewiesen und

142 Zum Fluchtort für die höheren Behörden und Kassen der Provinz Schleswig-Holstein war bei akuter Gefahr Magdeburg bestimmt worden. GStA PK, I. HA, Rep. 77 Ministerium des Innern, Tit. $332^{\text {bb }}$, Nr. 4, Bd 2, Kiel, 21.7.1870.

143 Ebd., Tit. 330 ${ }^{\mathrm{a}}$, Nr. 22, fol. 84, 123, 128, 133 und fol. 136.

144 Ebd., Nr. 18, Bd 1, Königsberg, 13.8.1870.

145 Ebd., Berlin, 7.9.1870.

146 Ebd., Berlin, 28.1.1883. 
folglich eine gewisse Verpflichtung zur Fürsorge anerkannt. Konkrete Maßnahmen zur Eindämmung der tradierten militärischen Ansprüche unterblieben jedoch; statt dessen bestätigte Ministerpräsident von Caprivi (zugleich Reichskanzler) im Frühsommer 1891 die Gültigkeit der bestehenden Vorschriften, indem er die Befehlshaber eingeschlossener Festungen für »vollständig souverain $\aleph^{147}$ erklärte und hiermit zugleich als ehemaliger General die geringe militärische Neigung zur Abänderung der umstrittenen, längst unzeitgemäßen Ermächtigung zu erkennen gab. Die Vorrechte blieben also gewahrt; sie waren noch bei Ausbruch des Ersten Weltkriegs maßgebend, wie Überlegungen zur Ausweisung von 60000 Einwohnern aus der Festung Posen (gut 38\% der Städter) und 6000 aus Thorn (13\%) im Spätsommer und Herbst 1914 beim Vorstoß russischer Truppen in die Ostprovinzen belegen $^{148}$.

147 Die Protokolle des Preußischen Staatsministeriums 1817-1934/38, Bd 106, fol. 24 (Mikrofiches-Ausgabe). Vgl. auch Böckling, Frauen (wie Anm. 3), S. 64.

148 GStA PK, I. HA, Rep. 77 Ministerium des Innern, Tit. 332v , Nr. 100, Bd 1, Posen, 28.8.1914, siehe ferner Posen, 30.10.1914; Berlin, 14.11.1914. 